\title{
45th International Colloquium on Automata, Languages, and Programming
}

ICALP 2018, Prague, Czech Republic, July 9-13, 2018

Edited by

loannis Chatzigiannakis

Christos Kaklamanis

Dániel Marx

Donald Sannella

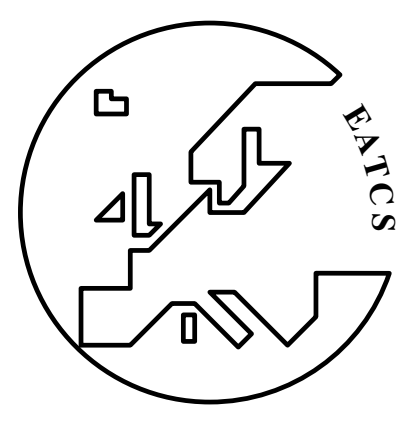




\section{Editors}

loannis Chatzigiannakis

Department of Computer, Control, and Management Engineering

Sapienza University of Rome

ichatz@dis.uniroma1.it

Dániel Marx

Institute for Computer Science and Control

Hungarian Academy of Sciences

dmarx@cs.bme.hu
Christos Kaklamanis

Department of Computer Engineering and Informatics University of Patras and

CTI "Diophantus"

ckakl@cti.gr

Donald Sannella

School of Informatics

University of Edinburgh

dts@inf.ed.ac.uk

ACM Classification 2012

Theory of computation

\section{ISBN 978-3-95977-076-7}

Published online and open access by

Schloss Dagstuhl - Leibniz-Zentrum für Informatik GmbH, Dagstuhl Publishing, Saarbrücken/Wadern, Germany. Online available at http://www.dagstuhl.de/dagpub/978-3-95977-076-7.

Publication date

July, 2018

Bibliographic information published by the Deutsche Nationalbibliothek

The Deutsche Nationalbibliothek lists this publication in the Deutsche Nationalbibliografie; detailed bibliographic data are available in the Internet at http://dnb.d-nb.de.

\section{License}

This work is licensed under a Creative Commons Attribution 3.0 Unported license (CC-BY 3.0): http://creativecommons.org/licenses/by/3.0/legalcode.

In brief, this license authorizes each and everybody to share (to copy, distribute and transmit) the work under the following conditions, without impairing or restricting the authors' moral rights:

- Attribution: The work must be attributed to its authors.

The copyright is retained by the corresponding authors.

Digital Object Identifier: 10.4230/LIPIcs.ICALP.2018.0

ISBN 978-3-95977-076-7

ISSN 1868-8969 http://www.dagstuhl.de/lipics 


\section{LIPIcs - Leibniz International Proceedings in Informatics}

LIPIcs is a series of high-quality conference proceedings across all fields in informatics. LIPIcs volumes are published according to the principle of Open Access, i.e., they are available online and free of charge.

\section{Editorial Board}

- Luca Aceto (Chair, Gran Sasso Science Institute and Reykjavik University)

- Susanne Albers (TU München)

- Chris Hankin (Imperial College London)

- Deepak Kapur (University of New Mexico)

- Michael Mitzenmacher (Harvard University)

- Madhavan Mukund (Chennai Mathematical Institute)

- Anca Muscholl (University Bordeaux)

- Catuscia Palamidessi (INRIA)

- Raimund Seidel (Saarland University and Schloss Dagstuhl - Leibniz-Zentrum für Informatik)

- Thomas Schwentick (TU Dortmund)

- Reinhard Wilhelm (Saarland University)

\section{ISSN 1868-8969}

\section{http://www.dagstuhl.de/lipics}





\section{Contents}

Preface

Ioannis Chatzigiannakis, Christos Kaklamanis, Dániel Marx, and Donald Sannella 0:xv-0:xvi Organization

0:xvii-0:xxv

List of Authors

0:xxvii-0:xlvii

\section{Invited Papers}

Consistent Distributed Memory Services: Resilience and Efficiency

Theophanis Hadjistasi and Alexander A. Schwarzmann

Sparsity - an Algorithmic Perspective

Jaroslav Ně̌etřil . .

Probability Theory from a Programming Perspective

Sam Staton

Lower Bounds by Algorithm Design: A Progress Report

Richard Ryan Williams

\section{Track A: Algorithms, Complexity, and Games}

Power of $d$ Choices with Simple Tabulation Anders Aamand, Mathias Bak Tejs Knudsen, and Mikkel Thorup

One-Way Trail Orientations Anders Aamand, Niklas Hjuler, Jacob Holm, and Eva Rotenberg

Dynamic Matching: Reducing Integral Algorithms to Approximately-Maximal

Fractional Algorithms

Moab Arar, Shiri Chechik, Sarel Cohen, Cliff Stein, and David Wajc

Tighter Connections Between Formula-SAT and Shaving Logs Amir Abboud and Karl Bringmann

New Approximation Algorithms for $(1,2)$-TSP Anna Adamaszek, Matthias Mnich, and Katarzyna Paluch

Union of Hypercubes and 3D Minkowski Sums with Random Sizes

Pankaj K. Agarwal, Haim Kaplan, and Micha Sharir

Noise-Tolerant Testing of High Entanglement of Formation Rotem Arnon-Friedman and Henry Yuen

A Complete Dichotomy for Complex-Valued Holant ${ }^{\mathrm{c}}$ Miriam Backens

45th International Colloquium on Automata, Languages, and Programming (ICALP 2018).

Editors: Ioannis Chatzigiannakis, Christos Kaklamanis, Dániel Marx, and Donald Sannella Leibniz International Proceedings in Informatics

IPICS Schloss Dagstuhl - Leibniz-Zentrum für Informatik, Dagstuhl Publishing, Germany 
Tight Bounds on Online Checkpointing Algorithms Achiya Bar-On, Itai Dinur, Orr Dunkelman, Rani Hod, Nathan Keller, Eyal Ronen, and Adi Shamir

Fast Reed-Solomon Interactive Oracle Proofs of Proximity

Eli Ben-Sasson, Iddo Bentov, Yinon Horesh, and Michael Riabzev ...

NP-Hardness of Coloring 2-Colorable Hypergraph with Poly-Logarithmically

Many Colors

Amey Bhangale

Sublinear Algorithms for MAXCUT and Correlation Clustering

Aditya Bhaskara, Samira Daruki, and Suresh Venkatasubramanian

Parameterized Intractability of Even Set and Shortest Vector Problem from

Gap-ETH

Arnab Bhattacharyya, Suprovat Ghoshal, Karthik C. S., and Pasin Manurangsi ..

Rollercoasters and Caterpillars

Therese Biedl, Ahmad Biniaz, Robert Cummings, Anna Lubiw, Florin Manea,

Dirk Nowotka, and Jeffrey Shallit

$18: 1-18: 15$

New algorithms for Steiner tree reoptimization

Davide Bilò

Efficient Shortest Paths in Scale-Free Networks with Underlying Hyperbolic

Geometry

Thomas Bläsius, Cedric Freiberger, Tobias Friedrich, Maximilian Katzmann,

Felix Montenegro-Retana, and Marianne Thieffry

Approximate Convex Hull of Data Streams

Avrim Blum, Vladimir Braverman, Ananya Kumar, Harry Lang, and Lin F. Yang

Small Bias Requires Large Formulas

Andrej Bogdanov

Geodesic Obstacle Representation of Graphs

Prosenjit Bose, Paz Carmi, Vida Dujmovic, Saeed Mehrabi, Fabrizio Montecchiani,

Pat Morin, and Luis Fernando Schultz Xavier da Silveira

The Bottleneck Complexity of Secure Multiparty Computation

Elette Boyle, Abhishek Jain, Manoj Prabhakaran, and Ching-Hua Yu

$24: 1-24: 16$

Revisiting Frequency Moment Estimation in Random Order Streams

Vladimir Braverman, Emanuele Viola, David P. Woodruff, and Lin F. Yang .....

$25: 1-25: 14$

Proportional Approval Voting, Harmonic k-median, and Negative Association

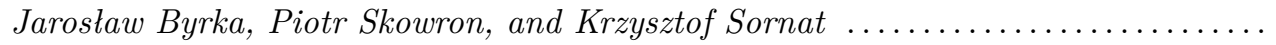

Fine-Grained Derandomization: From Problem-Centric to Resource-Centric

Complexity

Marco L. Carmosino, Russell Impagliazzo, and Manuel Sabin

Ranking with Fairness Constraints

L. Elisa Celis, Damian Straszak, and Nisheeth K. Vishnoi

$28: 1-28: 15$ 
Interpolating between $k$-Median and $k$-Center: Approximation Algorithms for Ordered $k$-Median

Deeparnab Chakrabarty and Chaitanya Swamy

29:1-29:14

Generalized Center Problems with Outliers

Deeparnab Chakrabarty and Maryam Negahbani

Orthogonal Point Location and Rectangle Stabbing Queries in 3-d

Timothy M. Chan, Yakov Nekrich, Saladi Rahul, and Konstantinos Tsakalidis ....

Spanning Tree Congestion and Computation of Generalized Győri-Lovász

Partition

L. Sunil Chandran, Yun Kuen Cheung, and Davis Issac

Fully Dynamic Almost-Maximal Matching: Breaking the Polynomial Worst-Case

Time Barrier

Moses Charikar and Shay Solomon

$33: 1-33: 14$

On Estimating Edit Distance: Alignment, Dimension Reduction, and Embeddings Moses Charikar, Ofir Geri, Michael P. Kim, and William Kuszmaul

How Hard Is It to Satisfy (Almost) All Roommates?

Jiehua Chen, Danny Hermelin, Manuel Sorge, and Harel Yedidsion

$35: 1-35: 15$

A Quadratic Size-Hierarchy Theorem for Small-Depth Multilinear Formulas

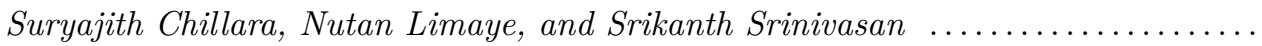

$36: 1-36: 13$

Restricted Max-Min Fair Allocation

Siu-Wing Cheng and Yuchen Mao

Improved Approximation for Node-Disjoint Paths in Grids with Sources on the

Boundary Julia Chuzhoy, David H. K. Kim, and Rachit Nimavat

Optimal Hashing in External Memory Alex Conway, Martín Farach-Colton, and Philip Shilane

Lovász Meets Weisfeiler and Leman Holger Dell, Martin Grohe, and Gaurav Rattan

Sample-Optimal Identity Testing with High Probability Ilias Diakonikolas, Themis Gouleakis, John Peebles, and Eric Price

Approximating All-Pair Bounded-Leg Shortest Path and APSP-AF in

Truly-Subcubic Time

Ran Duan and Hanlin Ren

Single-Source Bottleneck Path Algorithm Faster than Sorting for Sparse Graphs

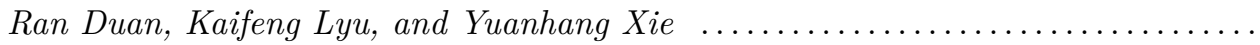

Improved Time Bounds for All Pairs Non-decreasing Paths in General Digraphs Ran Duan, Yong Gu, and Le Zhang

Edit Distance between Unrooted Trees in Cubic Time Bartłomiej Dudek and Pawet Gawrychowski 
A Note on Two-Colorability of Nonuniform Hypergraphs

Lech Duraj, Grzegorz Gutowski, and Jakub Kozik

$46: 1-46: 13$

Additive Non-Approximability of Chromatic Number in Proper Minor-Closed

Classes

Zdeněk Dvořák and Ken-ichi Kawarabayashi

How to Navigate Through Obstacles?

Eduard Eiben and Iyad Kanj

Faster Algorithms for Integer Programs with Block Structure

Friedrich Eisenbrand, Christoph Hunkenschröder, and Kim-Manuel Klein

On the Probe Complexity of Local Computation Algorithms

Uriel Feige, Boaz Patt-Shamir, and Shai Vardi

Fully-Dynamic Bin Packing with Little Repacking Björn Feldkord, Matthias Feldotto, Anupam Gupta, Guru Guruganesh, Amit Kumar, Sören Riechers, and David Wajc

A Sublinear Tester for Outerplanarity (and Other Forbidden Minors) With

One-Sided Error

Hendrik Fichtenberger, Reut Levi, Yadu Vasudev, and Maximilian Wötzel

$52: 1-52: 14$

Parameterized Low-Rank Binary Matrix Approximation

Fedor V. Fomin, Petr A. Golovach, and Fahad Panolan

$53: 1-53: 16$

Towards Blackbox Identity Testing of Log-Variate Circuits

Michael A. Forbes, Sumanta Ghosh, and Nitin Saxena

Finding Cliques in Social Networks: A New Distribution-Free Model

Jacob Fox, Tim Roughgarden, C. Seshadhri, Fan Wei, and Nicole Wein

A PTAS for a Class of Stochastic Dynamic Programs

Hao Fu, Jian Li, and Pan Xu

Semi-Supervised Algorithms for Approximately Optimal and Accurate Clustering

Buddhima Gamlath, Sangxia Huang, and Ola Svensson

High Probability Frequency Moment Sketches

Sumit Ganguly and David P. Woodruff

58:1-58:15

Quasi-PTAS for Scheduling with Precedences using LP Hierarchies

Shashwat Garg

$59: 1-59: 13$

ARRIVAL: Next Stop in CLS

Bernd Gärtner, Thomas Dueholm Hansen, Pavel Hubáček, Karel Král,

Hagar Mosaad, and Veronika Slívová

Improved Bounds for Shortest Paths in Dense Distance Graphs

Pawet Gawrychowski and Adam Karczmarz

Towards Unified Approximate Pattern Matching for Hamming and $L_{1}$ Distance

Pawel Gawrychowski and Przemysław Uznański

A Faster Construction of Greedy Consensus Trees

Pawel Gawrychowski, Gad M. Landau, Wing-Kin Sung, and Oren Weimann ..... 
A Faster FPTAS for \#Knapsack Pawet Gawrychowski, Liran Markin, and Oren Weimann

Towards Optimal Approximate Streaming Pattern Matching by Matching

Multiple Patterns in Multiple Streams

Shay Golan, Tsvi Kopelowitz, and Ely Porat

Gray Codes and Symmetric Chains

Petr Gregor, Sven Jäger, Torsten Mütze, Joe Sawada, and Kaja Wille

An Improved Isomorphism Test for Bounded-Tree-Width Graphs

Martin Grohe, Daniel Neuen, Pascal Schweitzer, and Daniel Wiebking ...

67:1-67:14

A Polynomial-Time Approximation Algorithm for All-Terminal Network

Reliability

Heng Guo and Mark Jerrum

68:1-68:12

Perfect Simulation of the Hard Disks Model by Partial Rejection Sampling Heng Guo and Mark Jerrum

Non-Preemptive Flow-Time Minimization via Rejections Anupam Gupta, Amit Kumar, and Jason Li

70:1-70:13

Maximizing Profit with Convex Costs in the Random-order Model Anupam Gupta, Ruta Mehta, and Marco Molinaro

Generic Single Edge Fault Tolerant Exact Distance Oracle Manoj Gupta and Aditi Singh

An Exponential Separation Between MA and AM Proofs of Proximity Tom Gur, Yang P. Liu, and Ron D. Rothblum

Isolating a Vertex via Lattices: Polytopes with Totally Unimodular Faces Rohit Gurjar, Thomas Thierauf, and Nisheeth K. Vishnoi

Synchronization Strings: Channel Simulations and Interactive Coding for Insertions and Deletions Bernhard Haeupler, Amirbehshad Shahrasbi, and Ellen Vitercik

Synchronization Strings: List Decoding for Insertions and Deletions Bernhard Haeupler, Amirbehshad Shahrasbi, and Madhu Sudan

Approximate Sparse Linear Regression Sariel Har-Peled, Piotr Indyk, and Sepideh Mahabadi

A Polynomial Time Algorithm to Compute Geodesics in CAT(0) Cubical Complexes

Koyo Hayashi

Online Vertex-Weighted Bipartite Matching: Beating $1-\frac{1}{e}$ with Random Arrivals Zhiyi Huang, Zhihao Gavin Tang, Xiaowei Wu, and Yuhao Zhang

Finding Branch-Decompositions of Matroids, Hypergraphs, and More Jisu Jeong, Eun Jung Kim, and Sang-il Oum

Optimally Sorting Evolving Data Juan Jose Besa, William E. Devanny, David Eppstein, Michael T. Goodrich, and Timothy Johnson 
Generalized Comparison Trees for Point-Location Problems Daniel M. Kane, Shachar Lovett, and Shay Moran ..

Stabilizing Weighted Graphs

Zhuan Khye Koh and Laura Sanità

Spectrally Robust Graph Isomorphism

Alexandra Kolla, Ioannis Koutis, Vivek Madan, and Ali Kemal Sinop

A Parameterized Strongly Polynomial Algorithm for Block Structured Integer

Programs

Martin Koutecký, Asaf Levin, and Shmuel Onn

Finer Tight Bounds for Coloring on Clique-Width

Michael Lampis

A Centralized Local Algorithm for the Sparse Spanning Graph Problem

Christoph Lenzen and Reut Levi

Chain, Generalization of Covering Code, and Deterministic Algorithm for k-SAT Sixue Liu

Stable-Matching Voronoi Diagrams: Combinatorial Complexity and Algorithms Gill Barequet, David Eppstein, Michael T. Goodrich, and Nil Mamano

Improved Algorithms for Adaptive Compressed Sensing

Vasileios Nakos, Xiaofei Shi, David P. Woodruff, and Hongyang Zhang ...

Approximate Low-Weight Check Codes and Circuit Lower Bounds for Noisy

Ground States

Chinmay Nirkhe, Umesh Vazirani, and Henry Yuen

Fully Dynamic MIS in Uniformly Sparse Graphs

Krzysztof Onak, Baruch Schieber, Shay Solomon, and Nicole Wein ...

Strictly Balancing Matrices in Polynomial Time Using Osborne's Iteration

Rafail Ostrovsky, Yuval Rabani, and Arman Yousefi

Parameterized Algorithms for Zero Extension and Metric Labelling Problems Felix Reidl and Magnus Wahlström

An Operational Characterization of Mutual Information in Algorithmic Information Theory Andrei Romashchenko and Marius Zimand

Privacy Preserving Clustering with Constraints Clemens Rösner and Melanie Schmidt .

NC Algorithms for Weighted Planar Perfect Matching and Related Problems

Piotr Sankowski

Computing Tutte Paths

Andreas Schmid and Jens M. Schmidt

A New Approximation Guarantee for Monotone Submodular Function

Maximization via Discrete Convexity

Tasuku Soma and Yuichi Yoshida 
Ring Packing and Amortized FHEW Bootstrapping Daniele Miccianco and Jessica Sorrell 100:1-100:14

Semi-random Graphs with Planted Sparse Vertex Cuts: Algorithms for Exact and Approximate Recovery

Anand Louis and Rakesh Venkat

Load Thresholds for Cuckoo Hashing with Overlapping Blocks

Stefan Walzer

Brief Announcement: On Secure $m$-Party Computation, Commuting Permutation

Systems and Unassisted Non-Interactive MPC

Navneet Agarwal, Sanat Anand, and Manoj Prabhakaran

Brief Announcement: Characterizing Demand Graphs for (Fixed-Parameter)

Shallow-Light Steiner Network

Amy Babay, Michael Dinitz, and Zeyu Zhang

Brief Announcement: Zero-Knowledge Protocols for Search Problems Ben Berger and Zvika Brakerski

Brief Announcement: Relaxed Locally Correctable Codes in Computationally

Bounded Channels

Jeremiah Blocki, Venkata Gandikota, Elena Grigorescu, and Samson Zhou 106:1-106:4

Brief Announcement: Approximation Schemes for Geometric Coverage Problems Steven Chaplick, Minati De, Alexander Ravsky, and Joachim Spoerhase ....... 107:1-107:4

Brief Announcement: Bayesian Auctions with Efficient Queries Jing Chen, Bo Li, Yingkai Li, and Pinyan Lu

Brief Announcement: Hamming Distance Completeness and Sparse Matrix

Multiplication

Daniel Graf, Karim Labib, and Przemystaw Uznański

Brief Announcement: Treewidth Modulator: Emergency Exit for DFVS

Daniel Lokshtanov, M. S. Ramanujan, Saket Saurabh, Roohani Sharma, and Meirav Zehavi

Brief Announcement: Erasure-Resilience Versus Tolerance to Errors Sofya Raskhodnikova and Nithin Varma

Brief Announcement: Bounded-Degree Cut is Fixed-Parameter Tractable Mingyu Xiao and Hiroshi Nagamochi

\section{Track B: Logic, Semantics, Automata and Theory of Programming}

Almost Sure Productivity Alejandro Aguirre, Gilles Barthe, Justin Hsu, and Alexandra Silva

O-Minimal Invariants for Linear Loops

Shaull Almagor, Dmitry Chistikov, Joël Ouaknine, and James Worrell

Topological Sorting with Regular Constraints Antoine Amarilli and Charles Paperman 
On Zero-One and Convergence Laws for Graphs Embeddable on a Fixed Surface Albert Atserias, Stephan Kreutzer, and Marc Noy

Bisimulation Invariant Monadic-Second Order Logic in the Finite Achim Blumensath and Felix Wolf

Binary Reachability of Timed Pushdown Automata via Quantifier Elimination and Cyclic Order Atoms

Lorenzo Clemente and Stawomir Lasota

Unboundedness Problems for Languages of Vector Addition Systems

Wojciech Czerwinski, Piotr Hofman, and Georg Zetzsche

Reachability and Distances under Multiple Changes

Samir Datta, Anish Mukherjee, Nils Vortmeier, and Thomas Zeume

When is Containment Decidable for Probabilistic Automata?

Laure Daviaud, Marcin Jurdziński, Ranko Lazić, Filip Mazowiecki,

Guillermo A. Pérez, and James Worrell

On the Complexity of Infinite Advice Strings Gaëtan Douéneau-Tabot

Resynchronizing Classes of Word Relations María Emilia Descotte, Diego Figueira, and Gabriele Puppis

Reachability Switching Games John Fearnley, Martin Gairing, Matthias Mnich, and Rahul Savani

Costs and Rewards in Priced Timed Automata Martin Fränzle, Mahsa Shirmohammadi, Mani Swaminathan, and James Worrell 125:1-125:14

First-Order Interpretations of Bounded Expansion Classes

Jakub Gajarský, Stephan Kreutzer, Jaroslav Nešetřil, Patrice Ossona de Mendez, Michat Pilipczuk, Sebastian Siebertz, and Szymon Toruńczyk

Randomized Sliding Window Algorithms for Regular Languages Moses Ganardi, Danny Hucke, and Markus Lohrey

Aperiodic points in $\mathbb{Z}^{2}$-subshifts

Anael Grandjean, Benjamin Hellouin de Menibus, and Pascal Vanier

Semicomputable Geometry

Mathieu Hoyrup, Diego Nava Saucedo, and Don M. Stull

On Computing the Total Variation Distance of Hidden Markov Models

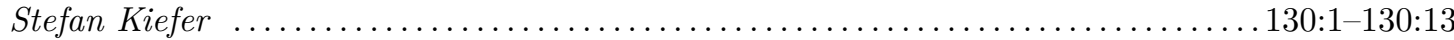

To Infinity and Beyond

Ines Klimann

On the Identity Problem for the Special Linear Group and the Heisenberg Group

Sang-Ki Ko, Reino Niskanen, and Igor Potapov

Gaifman Normal Forms for Counting Extensions of First-Order Logic Dietrich Kuske and Nicole Schweikardt 
Polynomial Vector Addition Systems With States Jérôme Leroux

Reducing CMSO Model Checking to Highly Connected Graphs Daniel Lokshtanov, M. S. Ramanujan, Saket Saurabh, and Meirav Zehavi

An Optimal Bound on the Solution Sets of One-Variable Word Equations and its

Consequences

Dirk Nowotka and Aleksi Saarela

Separating Without Any Ambiguity

Thomas Place and Marc Zeitoun

A Superpolynomial Lower Bound for the Size of Non-Deterministic Complement of an Unambiguous Automaton

Mikhail Raskin

The Isomorphism Problem for Finite Extensions of Free Groups Is In PSPACE Géraud Sénizergues and Armin Weiß

Unambiguous Languages Exhaust the Index Hierarchy Michat Skrzypczak

The Beta-Bernoulli process and algebraic effects

Sam Staton, Dario Stein, Hongseok Yang, Nathanael L. Ackerman,

Cameron E. Freer, and Daniel M. Roy

Uniformization Problems for Synchronizations of Automatic Relations on Words

Sarah Winter

\section{Track C: Foundations of Networked Computation: Models, Algorithms, and Information Management}

Congestion-Free Rerouting of Flows on DAGs Saeed Akhoondian Amiri, Szymon Dudycz, Stefan Schmid, and Sebastian Wiederrecht

Practical and Provably Secure Onion Routing Megumi Ando, Anna Lysyanskaya, and Eli Upfal

Resolving SINR Queries in a Dynamic Setting Boris Aronov, Gali Bar-On, and Matthew J. Katz

Uniform Mixed Equilibria in Network Congestion Games with Link Failures Vittorio Bilò, Luca Moscardelli, and Cosimo Vinci

Byzantine Gathering in Polynomial Time

Sébastien Bouchard, Yoann Dieudonné, and Anissa Lamani

Temporal Vertex Cover with a Sliding Time Window Eleni C. Akrida, George B. Mertzios, Paul G. Spirakis, and Viktor Zamaraev ... 148:1-148:14

On the Complexity of Sampling Vertices Uniformly from a Graph Flavio Chierichetti and Shahrzad Haddadan 
The Price of Stability of Weighted Congestion Games

George Christodoulou, Martin Gairing, Yiannis Giannakopoulos, and

Paul G. Spirakis

Demand-Independent Optimal Tolls

Riccardo Colini-Baldeschi, Max Klimm, and Marco Scarsini ...

$151: 1-151: 14$

Greedy Algorithms for Online Survivable Network Design

Sina Dehghani, Soheil Ehsani, MohammadTaghi Hajiaghayi, Vahid Liaghat, and

Saeed Seddighin

Algorithms for Noisy Broadcast with Erasures

Ofer Grossman, Bernhard Haeupler, and Sidhanth Mohanty

Efficient Black-Box Reductions for Separable Cost Sharing

Tobias Harks, Martin Hoefer, Anja Huber, and Manuel Surek

Price of Anarchy for Mechanisms with Risk-Averse Agents

Thomas Kesselheim and Bojana Kodric

Polynomial Counting in Anonymous Dynamic Networks with Applications to

Anonymous Dynamic Algebraic Computations

Dariusz R. Kowalski and Miguel A. Mosteiro

$156: 1-156: 14$

The Unfortunate-Flow Problem

Orna Kupferman and Gal Vardi

Spanning Trees With Edge Conflicts and Wireless Connectivity

Magnús M. Halldórsson, Guy Kortsarz, Pradipta Mitra, and Tigran Tonoyan ....158:1-158:15

Eigenvector Computation and Community Detection in Asynchronous Gossip

Models

Frederik Mallmann-Trenn, Cameron Musco, and Christopher Musco

$(\Delta+1)$ Coloring in the Congested Clique Model

Merav Parter

CacheShuffle: A Family of Oblivious Shuffles Sarvar Patel, Giuseppe Persiano, and Kevin Yeo

Brief Announcement: MapReduce Algorithms for Massive Trees MohammadHossein Bateni, Soheil Behnezhad, Mahsa Derakhshan, MohammadTaghi Hajiaghayi, and Vahab Mirrokni

Brief Announcement: Give Me Some Slack: Efficient Network Measurements Ran Ben Basat, Gil Einziger, and Roy Friedman ....................... 163:1-163:5

Brief Announcement: Towards an Abstract Model of User Retention Dynamics Eli Ben-Sasson and Eden Saig

Brief Announcement: Energy Constrained Depth First Search Shantanu Das, Dariusz Dereniowski, and Przemystaw Uznański $165: 1-165: 5$ 


\section{Preface}

This volume contains the papers presented at ICALP 2018, the 45th edition of the International Colloquium on Automata, Languages and Programming, held in Prague, Czech Republic during July 9-13, 2018. ICALP is a series of annual conferences of the European Association for Theoretical Computer Science (EATCS), which first took place in 1972. This year, the ICALP program consisted of three tracks:

- Track A: Algorithms, Complexity, and Games,

- Track B: Logic, Semantics, Automata and Theory of Programming,

- Track C: Foundations of Networked Computation: Models, Algorithms, and Information Management.

In response to the call for papers, a total 502 submissions were received: 346 for track $\mathrm{A}$, 96 for track B, and 60 for track C. Each submission was assigned to at least three Program Committee members, aided by many subreviewers. Out of these, the committee decided to accept 147 papers for inclusion in the scientific program: 98 papers for Track A, 30 for Track B, and 19 for Track C. The selection was made by the Program Committees based on originality, quality, and relevance to theoretical computer science. The quality of the manuscripts was very high, and many deserving papers could not be selected.

This year ICALP also solicitated brief announcements of work in progress with substantial interest for the community. In total 14 brief announcements were accepted for publication: 10 for Track A and 4 for Track C. The selection of the brief announcements was made by the Program Committees.

The EATCS sponsored awards for both a best paper and a best student paper for each of the three tracks, selected by the Program Committees.

The best paper awards were given to the following papers:

- Track A: Heng Guo and Mark Jerrum. "A polynomial-time approximation algorithm for all-terminal network reliability".

- Track B: Dirk Nowotka and Aleksi Saarela. "An optimal bound on the solution sets of one-variable word equations and its consequences".

- Track C: Dariusz Kowalski and Miguel A. Mosteiro. "Polynomial Counting in Anonymous Dynamic Networks with Applications to Anonymous Dynamic Algebraic Computations".

The best student paper awards, for papers that are solely authored by students, were given to the following papers:

- Track A: Shashwat Garg. "Quasi-PTAS for Scheduling with Precedences using LP Hierarchies".

- Track B: Sarah Winter. "Uniformization problems for synchronizations of automatic relations on words".

Apart from the contributed talks and the brief announcements, ICALP 2018 included invited presentations by Jaroslav Nešetřil, Alexander Schwarzmann, Sam Staton and Ryan Williams. This volume of the proceedings contains all contributed papers and brief announcements presented at the conference together with the papers and abstracts of the invited speakers.

The program of ICALP 2018 also included presentation of the EATCS Award 2018 to Noam Nisan, the Gödel Prize 2018 to Oded Regev, the Presburger Award 2018 to Aleksander

45th International Colloquium on Automata, Languages, and Programming (ICALP 2018).

Editors: Ioannis Chatzigiannakis, Christos Kaklamanis, Dániel Marx, and Donald Sannella Leibniz International Proceedings in Informatics

LI PICS Schloss Dagstuhl - Leibniz-Zentrum für Informatik, Dagstuhl Publishing, Germany 
Mądry, and the EATCS Distinguished Dissertation Award to Bas Ketsman, Ilya Razenshteyn and Aviad Rubinstein.

The program also included a memorial session for Maurice Nivat, the founder of ICALP and EATCS, who passed away in September 2017.

Six satellite events of ICALP were held on 9 July, 2018:

- Modern Online Algorithms (MOLI)

- Game Solving: Theory and Practice

- Parameterized Approximation Algorithms Workshop (PAAW)

- Infinity

- Algorithmic Aspects of Temporal Graphs

- Constrained Recognition Problems

The Summer School on Algorithms and Lower Bounds was organized immediately before ICALP during 6-9 July, 2018, with a follow-up workshop on Monday afternoon. The workshop was a satellite ICALP workshop devoted to presentations by selected participants of the school.

The Summer School on Discrete Mathematics was organized after the conference during 16-20 July, 2018. The event was organized by the Institute of Mathematics of the Czech Academy of Sciences and the Computer Science Institute of Charles University.

We wish to thank all authors who submitted extended abstracts for consideration, the Program Committees for their scholarly effort, and all referees who assisted the Program Committees in the evaluation process. We are also grateful to Anna Kotěšovcová from CONFORG and to Jiří Sgall, Andreas Emil Feldmann, Tomáš Masařík, Michal Opler, Jiř́ Fiala and Jan Musílek and all the support staff of the Organizing Committee from Charles University for organizing ICALP 2018.

We are grateful for generous support from AVAST and RSJ companies which included both travel grants for young women researchers and students and a direct support of the conference. We thank the School of Computer Science (Charles University, Faculty of Mathematics and Physics) and Center of Excellence - Institute for Theoretical Computer Science (project P202/12/G061 of Czech Science Foundation) for their support.

We would like to thank Jiří Sgall for his continuous support and Paul Spirakis, the president of EATCS, for his generous advice on the organization of the conference.

July 2018

Ioannis Chatzigiannakis

Christos Kaklamanis

Dániel Marx

Donald Sannella 


\section{Organization}

\section{Program Committee}

\section{Track A}

Dániel Marx
Alexandr Andoni
Nikhil Bansal
Markus Bläser
Glencora Borradaile
Sergio Cabello
Joseph Cheriyan
Leah Epstein
Samuel Fiorini
Craig Gentry
Kasper Green Larsen
Giuseppe F. Italiano
Bart M.P. Jansen
Petteri Kaski
Michal Koucký
Elias Koutsoupias
Robert Krauthgamer
Stephan Kreutzer
Troy Lee
Moshe Lewenstein
Monaldo Mastrolilli
Ankur Moitra
Seffi Naor
Seth Pettie
Michał Pilipczuk
Alon Rosen
Günter Rote
Barna Saha
Anastasios Sidiropoulos
Daniel Štefankovič
Maxim Sviridenko
Virginia Vassilevska Williams
Gerhard Woeginger
Ronald de Wolf
Stanislav Živný
Santion

Hungarian Academy of Sciences, Chair

Columbia University, USA

Eindhoven University of Technology, Netherlands

Saarland University, Germany

Oregon State University, USA

University of Ljubljana, Slovenia

University of Waterloo, Canada

University of Haifa, Israel

Université libre de Bruxelles, Belgium

IBM Research, USA

Aarhus University, Danmark

Università di Roma "Tor Vergata", Italy

Eindhoven University of Technology, Netherlands

Aalto University, Finland

Charles University, Czech Republic

Oxford, UK

Weizmann Institute, Israel

TU Berlin, Germany

Nanyang Technological University, Singapore

Bar-Ilan University, Israel

IDSIA, Switzerland

MIT, USA

Technion, Israel

University of Michigan, USA

University of Warsaw, Poland

Herzliya Interdisciplinary Center, Israel

Freie Universität Berlin, Germany

University of Massachusetts Amherst, USA

University of Illinois at Chicago, USA

University of Rochester, USA

Yahoo Research, USA

MIT, USA

RWTH Aachen, Germany

CWI and University of Amsterdam, Netherlands

Oxford, UK

\section{Track B}

Donald Sannella

Nathalie Bertrand

Mikołaj Bojańczyk

Udi Boker
Univ of Edinburgh, UK, Chair IRISA/INRIA Rennes, France Warsaw University, Poland Interdisciplinary Center Herzliya, Israel

45th International Colloquium on Automata, Languages, and Programming (ICALP 2018). Editors: Ioannis Chatzigiannakis, Christos Kaklamanis, Dániel Marx, and Donald Sannella Leibniz International Proceedings in Informatics

LI PICS Schloss Dagstuhl - Leibniz-Zentrum für Informatik, Dagstuhl Publishing, Germany 
Yuxin Deng

Floris Geerts

Dan Ghica

Alexey Gotsman

Jan Hoffmann

Naoki Kobayashi

Martin Lange

Dirk Pattinson

Femke van Raamsdonk

Jean-François Raskin

Vladimiro Sassone

Thomas Schwentick

Alex Simpson

Jiří Srba

Mirco Tribastone

Tomáš Vojnar

Igor Walukiewicz

Scott Weinstein

\section{Track C}

Christos Kaklamanis

Susanne Albers

Luca Becchetti

Ioannis Caragiannis

Andrea Clementi

Michele Flammini

Pierre Fraigniaud

Aristides Gionis

Sudipto Guha

Tomasz Jurdzinski

Evangelos Kranakis

Danny Krizanc

Katrina Ligett

Marios Mavronicolas

Kobbi Nissim

Marina Papatriantafilou

Andrzej Pelc

David Peleg

Geppino Pucci

Christian Scheideler

Roger Wattenhofer
East China Normal University, China

Univ Antwerp, Belgium

Univ Birmingham, UK

IMDEA, Spain

CMU, USA

Univ Tokyo, Japan

Univ Kassel, Germany

Australian National Univ, Australia

VU Amsterdam, Netherlands

Univ libre de Bruxelles, Belgium

Univ Southampton, UK

TU Dortmund, Germany

Univ Ljubljana, Slovenia

Aalborg Univ, Denmark

IMT Lucca, Italy

Brno Univ of Technology, Czech Republic

CNRS and Univ Bordeaux, France

Univ Pennsylvania, USA

CTI "Diophantus" and University of Patras, Greece, Chair

TU Munich, Germany

Sapienza University of Rome, Italy

University of Patras, Greece

University of Rome "Tor Vergata", Italy

Gran Sasso Sci Inst and Univ of L'Aquila, Italy

CNRS and Université Paris Diderot, France

Aalto University, Finland

University of Pennsylvania, USA

University of Wroclaw, Poland

Carleton University, Canada

Wesleyan University, USA

California Institute of Technology, USA and Hebrew University, Israel

University of Cyprus, Cyprus

Georgetown University, USA

Chalmers University of Technology, Sweden

Université du Québec en Outaouais, Canada

Weizmann Institute of Science, Israel

University of Padova, Italy

Paderborn University, Germany

ETH Zurich, Switzerland 


\section{Organizing Committee}

Jiří Sgall

Anna Kotěšovcová

Andreas Emil Feldmann

Tomáš Masařík

Michal Opler

Jiří Fiala

Jan Musílek

\section{Financial Sponsors}

Charles University, Czech Republic, Conference Chair CONFORG, Czech Republic

Charles University, Czech Republic Charles University, Czech Republic Charles University, Czech Republic Charles University, Czech Republic Charles University, Czech Republic
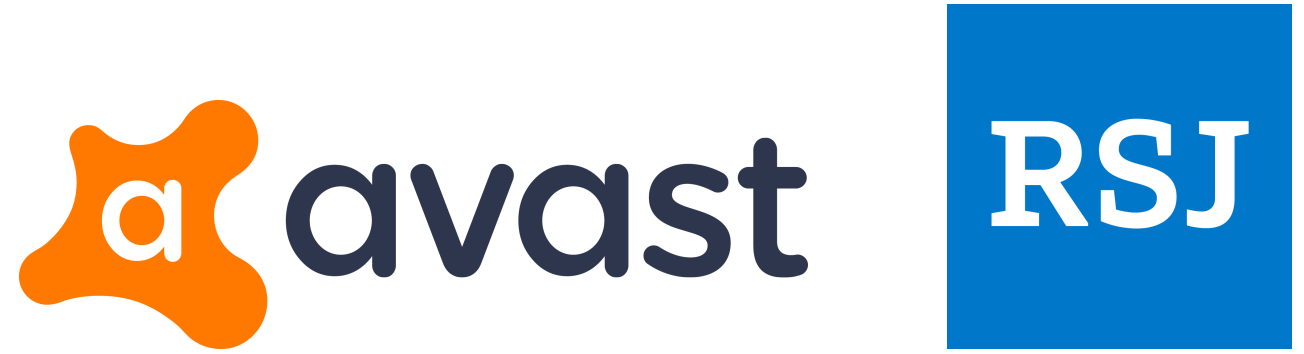


\section{Additional Reviewers}

Anders Aamand

Rupam Acharyya

Peyman Afshani

Saba Ahmadi

Shaull Almagor

Helmut Alt

Nima Anari

Itai Arad

Kazuyuki Asada

Cigdem Aslay

Chen Avin

Yakov Babichenko

Eric Balkanski

János Balogh

Bahareh Banyassady

Felix Baschenis

Paul Beame

Alexander Belov

Nikola Benes

Sebastian Berndt

Aditya Bhaskara

Philip Bille

Ahmad Biniaz

Jaroslaw Blasiok

Hans L. Bodlaender

Ralph Bottesch

Cornelius Brand

Karl Bringmann

Joshua Brody

Boris Bukh

Karthik C. S.

Arnaud Carayol

Matteo Ceccarello

Deeparnab Chakrabarty

Parinya Chalermsook

Timothy M. Chan

Yi-Jun Chang

Shiri Chechik

Xi Chen

Siu-Wing Cheng

Rajesh Chitnis

George Christodoulou

Raphaël Clifford

Edith Cohen

Thomas Colcombet
Mohammad Ali Abam

Anil Ada

Divesh Aggarwal

S. Akshay

Josh Alman

Joel Alwen

Leonardo Aniello

Myrto Arapinis

Gilad Asharov

Sepehr Assadi

Yossi Azar

Arturs Backurs

Marshall Ball

Stephanie Balzer

Amotz Bar-Noy

Mohammadhossein Bateni

Djamal Belazzougui

Omri Ben-Eliezer

Iddo Bentov

Aaron Bernstein

Sayan Bhattacharya

Davide Bilò

Nir Bitansky

Ivan Bliznets

Greg Bodwin

Patricia Bouyer

Vladimir Braverman

Sabine Broda

Vaclav Brozek

Sam Buss

Yang Cai

Clément Carbonnel

Keren Censor-Hillel

Diptarka Chakraborty

Jeremie Chalopin

Karthekeyan Chandrasekaran

Melissa Chase

Jiecao Chen

Yijia Chen

Victor Chepoi

Eden Chlamtac

Lorenzo Clemente

Adrien Le Coënt

Ilan Cohen

Denis Cornaz
Amir Abboud

Raghavendra Addanki

Manindra Agrawal

Xavier Allamigeon

Noga Alon

Andris Ambainis

Simon Apers

Srinivasan Arunachalam

Andrei Asinowski

Chen Attias

Haris Aziz

Saikrishna Badrinarayanan

Alkida Balliu

Jørgen Bang-Jensen

Ran Ben Basat

Tugkan Batu

Paul Bell

Souha Ben-Rayana

Suman Kalyan Bera

Ivona Bezakova

Marcin Bienkowski

Vittorio Bilò

Henrik Björklund

Achim Blumensath

Magnus Bordewich

Zvika Brakerski

Thomas Brihaye

Gerth Stølting Brodal

Kevin Buchin

Jaroslaw Byrka

Clément Canonne

Timothy Carpenter

Amit Chakrabarti

Sourav Chakraborty

T-H. Hubert Chan

Hsien-Chih Chang

Arkadev Chattopadhyay

Sitan Chen

Yu-Fang Chen

Dmitry Chistikov

Keerti Choudhary

Jonas Cleve

Avi Cohen

Vincent Cohen-Addad

Ágnes Cseh 
Radu Curticapean

Dana Dachman-Soled

Anupam Das

Anindya De

Stéphane Demri

David Doty

Adrian Dumitrescu

Rüdiger Ehlers

David Eisenstat

Jörg Endrullis

Guy Even

Yaron Fairstein

Bill Fefferman

Andreas Emil Feldmann

Henning Fernau

Emmanuel Filiot

Francesca Fiorenzi

Tamás Fleiner

Fedor Fomin

Tobias Friedrich

Vincent Froese

Benjamin Fuller

Andreas Galanis

Pierre Ganty

Olivier Gauwin

Samir Genaim

Shayan Oveis Gharan

Alex Gittens

Leslie Ann Goldberg

Isaac Goldstein

Alexander Golovnev

Thorsten Götte

Fabrizio Grandoni

Alex Bredariol Grilo

Roberto Grossi

Heng Guo

Anupam Gupta

Tom Gur

Torben Hagerup

Samuel Haney

Nicolas Hanusse

Tobias Harks

Tim Hartmann

Jacob Hendricks

Danny Hermelin

Denis Hirschfeldt

Dorit Hochbaum

Michael Hoffmann

Mathieu Hoyrup

Dawei Huang
Łukasz Czajka

Hadassa Daltrophe

Laure Daviaud

Éric Colin de Verdière

Michael Dinitz

Ran Duan

Romaric Dunignau

Eduard Eiben

Khaled Elbassioni

Matthias Englert

Esther Ezra

Jittat Fakcharoenphol

Uriel Feige

Michael Feldmann

Diego Figueira

Aris Filos-Ratsikas

Johannes Fischer

Krzysztof Fleszar

Casper Benjamin Freksen

Alan Frieze

Radoslav Fulek

Peter Gacs

Nicolas Gama

Sumegha Garg

Pawel Gawrychowski

Georgios Georgiadis

Panos Giannopoulos

Vasilis Gkatzelis

Paul Goldberg

Stefan Göller

Michael T. Goodrich

Lee-Ad Gottlieb

Roland Grappe

Martin Grohe

Luciano Gualà

Jiong Guo

Manoj Gupta

Venkatesan Guruswami

Mohammadtaghi Hajiaghayi

Kristoffer Arnsfelt Hansen

Sariel Har-Peled

David Harris

Carmit Hazay

Frédéric Herbreteau

John Hershberger

Petr Hlineny

Martin Hoefer

Stepan Holub

Justin Hsu

Zhiyi Huang
Artur Czumaj

Ankush Das

Anuj Dawar

Giorgio Delzanno

Michael Gene Dobbins

Vida Dujmovic

Martin Dyer

Friedrich Eisenbrand

Lior Eldar

Hossein Esfandiari

Yuri Faenza

Carlo Fantozzi

Moran Feldman

Stefan Felsner

Nathanaël Fijalkow

Arnold Filtser

Orr Fischer

Till Fluschnik

Dominik D. Freydenberger

Zachary Friggstad

Peter Fulla

Travis Gagie

Venkata Gandikota

Paul Gastin

Gilles Geeraerts

Loukas Georgiadis

Hugo Gimbert

Shay Golan

Elazar Goldenberg

Petr Golovach

Sivakanth Gopi

Themis Gouleakis

Alexander Grigoriev

Allan Grønlund

Bruno Guillon

Krystal Guo

Sushmita Gupta

Julian Gutierrez

Nir Halman

Thomas Dueholm Hansen

Tero Harju

Prahladh Harsha

Brett Hemenway

Kieran Herley

Hiroshi Hirai

Rebecca Hoberg

Frank Hoffmann

Stefan Hougardy

Chien-Chung Huang

Pavel Hubáček 
Vincent Hugot

Thore Husfeldt

Zvonko Iljazovic

Vincenzo Iovino

David Janin

Mark Jerrum

Shaofeng Jiang

Hossein Jowhari

Christos Kalaitzis

Lior Kamma

Iyad Kanj

Aikaterini Karanasiou

Zohar Karnin

Telikepalli Kavitha

Marcel Keller

Daniel Kernberger

Samir Khuller

Eunjung Kim

Philip Klein

Max Klimm

Jens Knoop

Tomasz Kociumaka

Christina Kolb

Spyros Kontogiannis

Guy Kortsarz

Jan Kratochvil

Jean Krivine

Amit Kumar

Marvin Künnemann

Thijs Laarhoven

Bundit Laekhanukit

Julien Lange

Sophie Laplante

Silvio Lattanzi

Euiwoong Lee

Ondrej Lengal

Peter Leupold

Maxwell Levit

Jason Li

Ray Li

Didier Lime

Nathan Lindzey

Christof Löding

Daniel Lokshtanov

Anand Louis

Pinyan $\mathrm{Lu}$

Vladimir Lysikov

Michael Mahoney

Yury Makarychev

David Manlove
Norbert Hundeshagen

Tony Huynh

Neil Immerman

Zahra Jafargholi

David N. Jansen

Artur Jeż

Adrian Johnstone

Chiraag Juvekar

Sagar Kale

Frank Kammer

Erez Kantor

Jarkko Kari

Takashi Katoh

Ken-Ichi Kawarabayashi

Hans Kellerer

Thomas Kesselheim

Stefan Kiefer

Sándor Kisfaludi-Bak

Robert Kleinberg

Jan Willem Klop

Dušan Knop

Ioannis Kokkinis

Balagopal Komarath

Tsvi Kopelowitz

Yiannis Koutis

Klaus Kriegel

Robert Kübler

Mrinal Kumar

Denis Kuperberg

Anthony Labarre

Michael Lampis

Stefan Langerman

Kim S. Larsen

Philip Lazos

Karoliina Lehtinen

Jérôme Leroux

Reut Levi

Avivit Levy

Jerry Li

Yuanzhi Li

Andrea Lincoln

Andre Linhares

Maarten Löffler

Federico Lombardi

Shachar Lovett

Jack H Lutz

Ramanujan M. S.

Hemanta Maji

Pasquale Malacaria

Giovanni Manzini
Christoph Hunkenschröder

John Iacono

Piotr Indyk

Ragesh Jaiswal

T.S. Jayram

Łukasz Jeż

Peter Jonsson

Volker Kaibel

Gautam Kamath

Daniel Kane

Michael Kapralov

Juha Kärkkäinen

Isabella Kaufmann

Steven Kelk

Dominik Kempa

Shahbaz Khan

Daniel Kifer

Kim-Manuel Klein

Boris Klemz

Katharina Klost

Yusuke Kobayashi

Sudeshna Kolay

Christian Konrad

Swastik Kopparty

Karel Král

S Krishna

Oliver Kullmann

Ravi Kumar

Salvatore La Torre

Arnaud Labourel

Patrick Landwehr

Elmar Langetepe

Sławomir Lasota

Hung Le

Steffen Lempp

Stefano Leucci

Asaf Levin

Nathan Lhote

Jian Li

Nutan Limaye

Steven Lindell

Quanquan Liu

Markus Lohrey

Julian Loss

Hsueh-I Lu

James F. Lynch

Mohammad Mahdian

Konstantin Makarychev

Andreas Maletti

Andrea Margheri 
Nicolas Markey

Tomas Masopust

Manuel Mauro

Andrew McGregor

Kurt Mehlhorn

Massimo Merro

Tom Meyerovitch

Marius Mikučionis

Carl Miller

Joseph Mitchell

Rolf H. Möhring

Cris Moore

Dana Moshkovitz

Wolfgang Mulzer

Cameron Musco

Daniel Nagaj

Guyslain Naves

Mark-Jan Nederhof

Ofer Neiman

Van Chan Ngo

Matthias Niewerth

Krzysztof Nowicki

Alexander Okhotin

Dennis Olivetti

Aurélien Ooms

Yota Otachi

Sang-Il Oum

Rasmus Pagh

Igor Pak

Gopal Pandurangan

Nikos Parotsidis

Francesco Pasquale

Lehilton L. C. Pedrosa

Richard Peng

Will Perkins

Andrea Pietracaprina

Jean-Eric Pin

Vladimir Podolskii

M. Praveen

Pavel Pudlak

Youming Qiao

Balaji Raghavachari

Govind Ramnarayan

Julian Rathke

Dror Rawitz

Vojtech Rehak

Bruce Richter

Liam Roditty

Dana Ron

Jurriaan Rot
Euripides Markou

Antonis Matakos

Richard Mayr

Moti Medina

Ruta Mehta

Julian Mestre

Theresa Migler-Vondollen

Martin Milanič

Tillmann Miltzow

Matthias Mnich

Tobias Mömke

Pat Morin

Elchanan Mossel

Kamesh Munagala

Christopher Musco

Viswanath Nagarajan

Amir Nayyeri

Jesper Nederlof

Adrian Neumann

Huy Nguyen

Filip Niksic

Marc Noy

Igor Carboni Oliveira

Feyishayo Olukoya

Sebastian Ordyniak

Joël Ouaknine

Megan Owen

Linda Pagli

Konstantinos Panagiotou

Debmalya Panigrahi

Merav Parter

Erik Paul

Chris Peikert

Guillermo Perez

Jeff Phillips

Oleg Pikhurko

Thomas Place

Ely Porat

Eric Price

Simon Puglisi

Daowen Qiu

Ajitha Rajan

Narad Rampersad

Gaurav Rattan

Jean-Florent Raymond

Daniel Reichman

Havana Rika

Heiko Röglin

Adi Rosén

Marc Roth
Francisco Martins

Bastien Maubert

Arya Mazumdar

Mohammad Syed Meesum

Manor Mendel

Pierre-étienne Meunier

Matúš Mihalák

Kevin Milans

Pranabendu Misra

Ali Mohades

Benjamin Moore

Ben Moseley

Shay Mozes

Ian Munro

Torsten Mütze

Anand Natarajan

Amir Nayyeri

Joe Neeman

Ilan Newman

Denis Nicole

Gali Noti

Timm Oertel

Rafael Oliveira

Krzysztof Onak

Mikhail Ostrovskii

Youssouf Oualhadj

Kenta Ozeki

Dominik Pajak

Anurag Pandey

Periklis Papakonstantinou

Anat Paskin-Cherniavsky

Subhabrata Paul

Pan Peng

Pablo Pérez-Lantero

Astrid Pieterse

Marcin Pilipczuk

Wojciech Plandowski

Gustavo Posta

Kirk Pruhs

Manish Purohit

Karin Quaas

Rajmohan Rajaraman

Sofya Raskhodnikova

Malin Rau

Ilya Razenshteyn

Ahmed Rezine

Andrej Risteski

Lars Rohwedder

Peter Rossmanith

Ron Rothblum 
Thomas Rothvoss

Michał Różański

Aleksi Saarela

Ken Sakayori

Laura Sanita

Rahul Santhanam

Jayalal Sarma

Srinivasa Rao Satti

Nitin Saurabh

Raghuvansh Saxena

Nadja Scharf

Georg Schnitger

Roy Schwartz

Elizabeth Scott

Danny Segev

Alexander Setzer

Don Sheehy

Tong-Wook Shinn

Rodrigo Silveira

Alistair Sinclair

Alexander Skopalik

Christian Sommer

Christopher Spinrath

Vijay Sridhar

Nikhil Srivastava

Damien Stehle

Noah Stephens-Davidowitz

Martin Sulzmann

Toshio Suzuki

Navid Talebanfard

Li-Yang Tan

Justin Thaler

Dimitrios Thilikos

Simone Tini

Patrick Totzke

Elias Tsakas

Takeshi Tsukada

Yuya Uezato

Ali Vakilian

Rob van Stee

Prashant Vasudevan

László A. Végh

Nikolay Vereshchagin

Cosimo Vinci

Nils Vortmeier

Magnus Wahlström

Johannes Waldmann

Yuyi Wang

Oren Weimann

Stefan Weltge
Reuben Rowe

Polina Rozenshtein

Sushant Sachdeva

Michael Saks

Piotr Sankowski

Ramprasad Saptharishi

Kanthi Sarpatwar

Ignasi Sau

Saket Saurabh

Guillaume Scerri

Sven Schewe

Tselil Schramm

Chris Schwiegelshohn

Adam Sealfon

Ilya Sergey

Asaf Shapira

Tetsuo Shibuya

Aaron Sidford

Francesco Silvestri

Kritika Singhal

Michał Skrzypczak

Fu Song

Sophie Spirkl

Akshayaram Srinivasan

B Srivathsan

Fabian Stehn

Sebastian Stiller

Scott Summers

Ola Svensson

Ohad Talmon

Jakub Tarnawski

Sharma V. Thankachan

Dilys Thomas

Andreas Tönnis

Henry Towsner

Max Tschaikowski

Iddo Tzameret

Seeun William Umboh

Leo van Iersel

Anke van Zuylen

Yann Vaxès

Santosh Vempala

José Verschae

Sundar Vishwanathan

Satyanarayana Vusirikala

Erik Waingarten

Erik Walsberg

Justin Ward

Omri Weinstein

Anthony Widjaja Lin
Eric Rowland

Atri Rudra

S. Cenk Sahinalp

Ario Salmasi

Ocan Sankur

Thatchaphol Saranurak

Tetsuya Sato

Thomas Sauerwald

Zdenek Sawa

Michael Schapira

Stefan Schmid

Dominique Schroeder

Giacomo Scornavacca

Erel Segal-Halevi

C. Seshadhri

Micha Sharir

Igor Shinkar

Sebastian Siebertz

Ryoma Sin'Ya

Rene Sitters

Shay Solomon

Aikaterini Sotiraki

Joachim Spoerhase

Aravind Srinivasan

Tatiana Starikovskaya

Florian Steinberg

Thomas Sturm

Aarthi Sundaram

Avishay Tal

Suguru Tamaki

Yael Tauman Kalai

Johan Thapper

Francesco Tiezzi

Tigran Tonoyan

Ohad Trabelsi

Philippas Tsigas

Marc Uetz

Rohit Vaish

Erik Jan van Leeuwen

Shai Vardi

Rahul Vaze

Carmine Ventre

Aravindan Vijayaraghavan

Jan Vondrak

Nikhil Vyas

David Wajc

Di Wang

Pascal Weil

Mathias Weller

Andreas Wiese 
Max Willert

Karl Wimmer

Dominik Wojtczak

James Worrell

Pei Wu

Ning Xie

Grigory Yaroslavtsev

Neal Young

Meirav Zehavi

Hanmeng Zhan

Baigong Zheng
Jack Williams

Sarah Winter

Bruno Woltzenlogel Paleo

Marcin Wrochna

Zhilin Wu

Lin F. Yang

Yitong Yin

Huacheng $\mathrm{Yu}$

Marc Zeitoun

Hantao Zhang

Vassilis Zikas
Ryan Williams

Philipp Woelfel

David P. Woodruff

David Wu

Christian Wulff-Nilsen

Mu Yang

Yuichi Yoshida

Or Zamir

Georg Zetzsche

Jie Zhang

Charilaos Zisopoulos 



\section{List of Authors}

Anders Aamand

BARC, University of Copenhagen,

Universitetsparken 1, Copenhagen, Denmark

aa@di.ku.dk

https://orcid.org/0000-0002-0402-0514

Amir Abboud

IBM Almaden Research Center, San Jose, USA

amir.abboud@ibm.com

Nathanael L. Ackerman

Harvard Univ

Anna Adamaszek

University of Copenhagen, Denmark

anad@di.ku.dk

Navneet Agarwal

Indian Institute of Technology Bombay

navneet@cse.iitb.ac.in

Pankaj K. Agarwal

Department of Computer Science, Duke

University, Durham, NC 27708, USA

pankaj@cs.duke.edu

Alejandro Aguirre

IMDEA Software Institute, Madrid, Spain

Saeed Akhoondian Amiri

Max-Planck Institute of Informatics,

Germany

samiri@mpi-inf .mpg.de

Eleni C. Akrida

Department of Computer Science, University of Liverpool, UK

Eleni.Akrida@liverpool.ac.uk

https://orcid.org/0000-0002-1126-1623

Shaull Almagor

Department of Computer Science, Oxford

University, UK

shaull.almagor@cs.ox.ac.uk

Antoine Amarilli

LTCI, Télécom ParisTech, Université

Paris-Saclay
Sanat Anand

Indian Institute of Technology Bombay

sanat@cse.iitb.ac.in

Megumi Ando

Computer Science Department, Brown

University, Providence, RI 02912 USA

mando@cs. brown.edu

Moab Arar

Tel Aviv University, Tel Aviv, Israel

Rotem Arnon-Friedman

ETH Zürich, Switzerland

rotema@itp.phys.ethz.ch

Boris Aronov

Department of Computer Science and

Engineering, Tandon School of Engineering, New York University, Brooklyn, NY 11201, USA

boris.aronov@nyu .edu

Albert Atserias

Universitat Politècnica de Catalunya,

Barcelona, atserias@cs.upc.edu

Amy Babay

Johns Hopkins University, Baltimore, MD,

USA

babay@cs . jhu .edu

Miriam Backens

Department of Computer Science, University

of Oxford, UK

miriam.backens@cs.ox.ac.uk

Achiya Bar-On

Department of Mathematics, Bar-Ilan

University, Ramat Gan, Israel

abo1000@gmail.com

Gali Bar-On

Department of Computer Science,

Ben-Gurion University of the Negev,

Beer-Sheva 84105, Israel

galibar@post.bgu.ac.il

45th International Colloquium on Automata, Languages, and Programming (ICALP 2018).

Editors: Ioannis Chatzigiannakis, Christos Kaklamanis, Dániel Marx, and Donald Sannella

Leibniz International Proceedings in Informatics

LIPICS Schloss Dagstuhl - Leibniz-Zentrum für Informatik, Dagstuhl Publishing, Germany 
Gill Barequet

Technion - Israel Inst. of Technology, Haifa,

Israel

barequet@cs.technion.ac.il

Gilles Barthe

IMDEA Software Institute, Madrid, Spain

MohammadHossein Bateni

Google Research, New York

Soheil Behnezhad

University of Maryland

Ran Ben Basat

Technion, Haifa, Israel

sran@cs.technion.ac.il

Eli Ben-Sasson

Department of Computer Science, Technion,

Haifa, Israel

eli@cs.technion.ac.il

https://orcid.org/0000-0002-0708-0483

Iddo Bentov

Cornell University, Ithaca, NY, USA

iddo333@gmail.com

Ben Berger

Weizmann Institute of Science, Rehovot, Israel

ben.berger@weizmann.ac.il

Juan Jose Besa

Dept. of Computer Science, Univ. of

California, Irvine, Irvine, CA 92697 USA

jjbesavi@uci.edu

https://orcid.org/0000-0002-5676-7011

Amey Bhangale

Weizmann Institute of Science, Rehovot, Israel

amey.bhangale@weizmann.ac.il

Aditya Bhaskara

School of Computing, University of Utah,

USA

bhaskara@cs.utah.edu

Arnab Bhattacharyya

Indian Institute of Science, Bangalore, India

arnabb@iisc.ac.in
Therese Biedl

School of Computer Science, University of

Waterloo, Canada

biedl@uwaterloo.ca

Davide Bilò

Department of Humanities and Social

Sciences, University of Sassari, Via Roma

151, 07100 Sassari (SS), Italy

davide.bilo@uniss.it

https://orcid.org/0000-0003-3169-4300

Vittorio Bilò

Department of Mathematics and Physics,

University of Salento, Lecce, Italy

vittorio.bilo@unisalento.it

Ahmad Biniaz

School of Computer Science, University of

Waterloo, Canada

ahmad.biniaz@gmail.com

Jeremiah Blocki

Department of Computer Science, Purdue University, West Lafayette, Indiana, USA jblocki@purdue.edu

Avrim Blum

TTI-Chicago, Chicago, United States avrim@ttic.edu

Achim Blumensath

Masaryk University Brno

blumens@fi.muni.cz

Thomas Bläsius

Hasso Plattner Institute, University of

Potsdam, Potsdam, Germany

thomas.blaesius@hpi.de

Andrej Bogdanov

Department of Computer Science and

Engineering and , Institute of Theoretical

Computer Science and Communications,

Chinese University of Hong Kong

andrejb@cse.cuhk.edu.hk

Prosenjit Bose

School of Computer Science, Carleton

University, Ottawa, Canada

jit@scs.carleton.ca 
Sébastien Bouchard

Sorbonne Universités, UPMC Univ Paris 06, CNRS, INRIA, LIP6 UMR 7606, Paris,

France

sebastien.bouchard@lip6.fr

Elette Boyle

IDC Herzliya

elette.boyle@idc.ac.il

Zvika Brakerski

Weizmann Institute of Science, Rehovot, Israel

zvika.brakerski@weizmann.ac.il

Vladimir Braverman

Johns Hopkins University, Baltimore, United

States

vova@cs.jhu.edu

Karl Bringmann

Max Planck Institute for Informatics,

Saarland Informatics Campus, Saarbrücken,

Germany

kbringma@mpi-inf .mpg.de

Jarosław Byrka

University of Wrocław, Wrocław, Poland

jby@cs.uni.wroc.pl

Mathias Bæk Tejs Knudsen

University of Copenhagen and Supwiz,

Copenhagen, Denmark

mathias@tejs.dk

https://orcid.org/0000-0001-5308-9609

Karthik C. S.

Weizmann Institute of Science, Rehovot, Israel

karthik.srikanta@weizmann.ac.il

Paz Carmi

Department of Computer Science, Ben-Gurion University of the Negev,

Beer-Sheva, Israel

carmip@cs.bgu.ac.il

Marco L. Carmosino

Department of Computer Science, University of California San Diego, La Jolla, CA, USA marco@ntime.org
L. Elisa Celis

École Polytechnique Fédérale de Lausanne (EPFL), Switzerland

Deeparnab Chakrabarty

Department of Computer Science,

Dartmouth College, 9 Maynard St, Hanover, $\mathrm{NH}, \mathrm{USA}$

deeparnab@dartmouth.edu

Timothy M. Chan

Dept. of Computer Science, University of Illinois at Urbana-Champaign, USA

tmc@illinois.edu

L. Sunil Chandran

Department of Computer Science and

Automation, Indian Institute of Science,

India

sunil@csa.iisc.ernet.in

Steven Chaplick

Lehrstuhl für Informatik I, Universität

Würzburg, Germany

steven. chaplick@uni-wuerzburg. de

https://orcid.org/0000-0003-3501-4608

Moses Charikar

Department of Computer Science, Stanford

University, Stanford, CA, USA

moses@cs.stanford.edu

Shiri Chechik

Tel Aviv University, Tel Aviv, Israel

Jiehua Chen

Ben-Gurion University of the Negev, Beer

Sheva, Israel

jiehua.chen2@gmail.com

Jing Chen

Department of Computer Science, Stony

Brook University, Stony Brook, NY 11794,

USA

jingchen@cs.stonybrook.edu

Siu-Wing Cheng

Department of Computer Science and

Engineering, HKUST, Hong Kong

scheng@cse.ust.hk

https://orcid.org/0000-0002-3557-9935 
Yun Kuen Cheung

Max Planck Institute for Informatics, Saarland Informatics Campus, Germany ycheung@mpi-inf .mpg . de https://orcid.org/0000-0002-9280-0149

Flavio Chierichetti

Dipartimento di Informatica, Sapienza

University, Rome, Italy

flavio@di.uniroma1.it

https://orcid.org/0000-0001-8261-9058

Suryajith Chillara

Department of CSE, IIT Bombay, Mumbai, India

suryajith@cse.iitb.ac.in

Dmitry Chistikov

Centre for Discrete Mathematics and its Applications (DIMAP) \&, Department of Computer Science, University of Warwick, UK

d.chistikov@warwick.ac.uk

George Christodoulou

Department of Computer Science, University

of Liverpool, Liverpool, UK

G.Christodoulou@liverpool.ac.uk

Julia Chuzhoy

Toyota Technological Institute at Chicago, 6045 S. Kenwood Ave., Chicago, Illinois 60637, USA

cjulia@ttic.edu

Lorenzo Clemente

University of Warsaw

clementelorenzo@gmail.com

https://orcid.org/0000-0003-0578-9103

Sarel Cohen

Tel Aviv University, Tel Aviv, Israel

Riccardo Colini-Baldeschi

Core Data Science Group, Facebook Inc., 1

Rathbone Place, London, W1T 1FB, UK

rickuz@fb.com

https://orcid.org/0000-0001-5739-1178

Alex Conway

Rutgers University, New Brunswick, NJ, USA

alexander. conway@rutgers . edu
Robert Cummings

School of Computer Science, University of

Waterloo, Canada

rcummings000@gmail.com

Wojciech Czerwiński

University of Warsaw, Poland

https ://orcid.org/0000-0002-6169-868X

Samira Daruki

Expedia Research, USA

sdaruki@expedia.com

Shantanu Das

LIS, Aix-Marseille University, University of Toulon, CNRS, Marseille, France

shantanu.das@lif.univ-mrs.fr

Samir Datta

Chennai Mathematical Institute \& UMI

ReLaX, Chennai, India

sdatta@cmi.ac.in

Laure Daviaud

University of Warwick, Coventry, UK

L.Daviaud@warwick.ac.uk

Minati De

Department of Mathematics, Indian Institute of Technology Delhi, India

minati@maths.iitd.ac.in

Sina Dehghani

University of Maryland, College Park, MD 20742, USA

Holger Dell

Saarland University and Cluster of

Excellence (MMCI), Saarbrücken, Germany

hdell@mmci.uni-saarland.de

https://orcid.org/0000-0001-8955-0786

Mahsa Derakhshan

University of Maryland

Dariusz Dereniowski

Faculty of Electronics, Telecommunications and Informatics, Gdańsk University of

Technology, Gdańsk, Poland

deren@eti.pg.edu.pl

https://orcid.org/0000-0003-4000-4818

María Emilia Descotte

LaBRI, Université de Bordeaux 
William E. Devanny

Dept. of Computer Science, Univ. of California, Irvine, Irvine, CA 92697 USA

wdevanny@uci.edu

Ilias Diakonikolas

USC, Los Angeles, CA, USA

diakonik@usc.edu

Yoann Dieudonné

Laboratoire MIS \& Université de Picardie

Jules Verne, Amiens, France

yoann.dieudonne@u-picardie.fr

Michael Dinitz

Johns Hopkins University, Baltimore, MD, USA

mdinitz@cs.jhu.edu

Itai Dinur

Computer Science Department, Ben-Gurion University, Beer Sheva, Israel

dinuri@cs.bgu.ac.il

Gaëtan Douéneau-Tabot

École Normale Supérieure Paris-Saclay,

Université Paris-Saclay, Cachan, France

gaetan.doueneau@ens-paris-saclay.fr

Ran Duan

Institute for Interdisciplinary Information

Sciences, Tsinghua University, China

duanran@mail.tsinghua.edu.cn

Bartłomiej Dudek

Institute of Computer Science, University of Wrocław, Poland

bartlomiej.dudek@cs.uni.wroc.pl

Szymon Dudycz

University of Wroclaw, Poland

szymon.dudycz@gmail.com

Vida Dujmovic

School of Computer Science and Electrical

Engineering, University of Ottawa, Ottawa,

Canada

vida.dujmovic@uottawa.ca

Orr Dunkelman

Computer Science Department, University of Haifa, Haifa, Israel

orrd@cs.haifa.ac.il

https://orcid.org/0000-0001-5799-2635
Lech Duraj

Theoretical Computer Science Department, Faculty of Mathematics and Computer Science, Jagiellonian University, Kraków, Poland

lech.duraj@uj.edu.pl

Zdeněk Dvořák

Charles University, Malostranske namesti 25, 11800 Prague, Czech Republic

rakdver@iuuk.mff.cuni.cz

https://orcid.org/0000-0002-8308-9746

Soheil Ehsani

University of Maryland, College Park, MD

20742, USA

Eduard Eiben

Department of Informatics, University of

Bergen, Bergen, Norway

eduard.eiben@uib.no

https://orcid.org/0000-0003-2628-3435

Gil Einziger

Nokia Bell Labs, Kfar Saba, Israel

gil.einziger@nokia.com

Friedrich Eisenbrand

EPFL, 1015 Lausanne, Switzerland

friedrich.eisenbrand@epf $1 . \mathrm{ch}$

David Eppstein

Dept. of Computer Science, Univ. of

California, Irvine, Irvine, CA 92697 USA

eppstein@uci.edu

Martín Farach-Colton

Rutgers University, New Brunswick, NJ, USA

farach@rutgers.edu

John Fearnley

University of Liverpool, UK

john.fearnley@liverpool.ac.uk

Uriel Feige

Weizmann Institute of Science, Rehovot, Israel

uriel.feige@weizmann.ac.il

Björn Feldkord

Paderborn University, Paderborn, Germany 
Matthias Feldotto

Paderborn University, Paderborn, Germany

Hendrik Fichtenberger

TU Dortmund, Dortmund, Germany

hendrik.fichtenberger@tu-dortmund.de

https://orcid.org/0000-0003-3246-5323

Diego Figueira

CNRS, LaBRI, Université de Bordeaux

Fedor V. Fomin

Department of Informatics, University of

Bergen, Norway

Fedor.Fomin@uib.no

https://orcid.org/0000-0003-1955-4612

Michael A. Forbes

University of Illinois at Urbana-Champaign, USA

miforbes@illinois.edu

Jacob Fox

Department of Mathematics, Stanford

University, Stanford, CA 94305, USA

jacobfox@stanford.edu

Cameron E. Freer

Borelian

Cedric Freiberger

Hasso Plattner Institute, University of

Potsdam, Potsdam, Germany

cedric.freiberger@student.hpi.de

Roy Friedman

Technion, Haifa, Israel

roy@cs.technion.ac.il

Tobias Friedrich

Hasso Plattner Institute, University of Potsdam, Potsdam, Germany

tobias.friedrich@hpi.de

Martin Fränzle

Department of Computing Science, University of Oldenburg, Germany

martin.fraenzle@informatik.

uni-oldenburg.de

$\mathrm{Hao} \mathrm{Fu}$

Institute for Interdisciplinary Information Sciences, Tsinghua University, Beijng, China fu-h130mails.tsinghua.edu.cn
Martin Gairing

Department of Computer Science, University of Liverpool, Liverpool, UK

gairing@liverpool.ac.uk

Jakub Gajarský

Technical University Berlin, Germany

Buddhima Gamlath

École Polytechnique Fédérale de Lausanne,

Lausanne, Switzerland

buddhima.gamlath@epfl.ch

Moses Ganardi

Universität Siegen, Germany

ganardi@eti.uni-siegen.de

Venkata Gandikota

Department of Computer Science, Johns

Hopkins University, Baltimore, Maryland,

USA

gv@jhu.edu

Sumit Ganguly

Indian Institute of Technology, Kanpur,

India

sganguly@cse.iitk.ac.in

Shashwat Garg

Eindhoven University of Technology,

Netherlands

s.garg@tue.nl

Paweł Gawrychowski

Institute of Computer Science, University of Wrocław, Poland

gawry@cs.uni.wroc.pl

Ofir Geri

Department of Computer Science, Stanford University, Stanford, CA, USA

ofirgeri@cs.stanford.edu

Sumanta Ghosh

Department of Computer Science, IIT

Kanpur, India

sumghosh@cse.iitk.ac.in

Suprovat Ghoshal

Indian Institute of Science, Bangalore, India suprovat@iisc.ac.in 
Yiannis Giannakopoulos

Department of Mathematics, TU Munich, Munich, Germany

Yiannis.Giannakopoulos@tum.de

https://orcid.org/0000-0003-2382-1779

\section{Shay Golan}

Bar Ilan University, Ramat Gan, Israel

golansh1@cs.biu.ac.il

Petr A. Golovach

Department of Informatics, University of Bergen, Norway

Petr.Golovach@uib.no

https://orcid.org/0000-0002-2619-2990

Michael T. Goodrich

Dept. of Computer Science, Univ. of California, Irvine, Irvine, CA 92697 USA goodrich@uci.edu

Themis Gouleakis

CSAIL,MIT, Cambridge, MA, USA

tgoule@mit.edu

Daniel Graf

Department of Computer Science, ETH

Zürich, Switzerland

daniel.graf@inf.ethz.ch

Anael Grandjean

Laboratoire d'Algorithmique, Complexité et Logique, Université Paris-Est Créteil, France anael.grandjean@u-pec.fr

Petr Gregor

Department of Theoretical Computer Science and Mathematical Logic, Charles University, Prague, Czech Republic gregor@ktiml.mff.cuni.cz

\section{Elena Grigorescu}

Department of Computer Science, Purdue University, West Lafayette, Indiana, USA elena-g@purdue.edu

\section{Martin Grohe}

RWTH Aachen University, Aachen, Germany grohe@informatik.rwth-aachen. de https://orcid.org/0000-0002-0292-9142
Ofer Grossman

EECS Departent, MIT, Cambridge, MA, USA

ofer.grossman@gmail.com

Yong $\mathrm{Gu}$

Institute for Interdisciplinary Information

Sciences, Tsinghua University, Beijing, China

guyong12@mails.tsinghua.edu.cn

Heng Guo

School of Informatics, University of

Edinburgh, Informatics Forum, Edinburgh, EH8 9AB, United Kingdom

hguo@inf.ed.ac.uk

https://orcid.org/0000-0001-8199-5596

Anupam Gupta

Carnegie Mellon University, Pittsburgh, USA

anupamg@cs.cmu.edu

Manoj Gupta

IIT Gandhinagar, Gandhinagar, India

gmanoj@iitgn.ac.in

Tom Gur

UC Berkeley, Berkeley, USA

tom.gur@berkeley . edu

Rohit Gurjar

California Institute of Technology, USA

Guru Guruganesh

Carnegie Mellon University, Pittsburgh, USA

Grzegorz Gutowski

Theoretical Computer Science Department, Faculty of Mathematics and Computer

Science, Jagiellonian University, Kraków,

Poland

grzegorz.gutowski@uj.edu.pl

https://orcid.org/0000-0003-3313-1237

Bernd Gärtner

Department of Computer Science, ETH

Zürich, Switzerland

gaertner@inf .ethz.ch

Shahrzad Haddadan

Dipartimento di Informatica, Sapienza

University, Rome, Italy

shahrzad.haddadan@uniroma1. it

https://orcid.org/0000-0002-7702-8250 
Theophanis Hadjistasi

University of Connecticut, Storrs CT, USA

theo@uconn.edu

Bernhard Haeupler

Computer Science Department, Carnegie

Mellon University, Pittsburgh, PA, USA

haeupler@cs.cmu . edu

MohammadTaghi Hajiaghayi

University of Maryland, College Park, MD

20742, USA

Magnús M. Halldórsson

School of Computer Science, Reykjavik

University, Iceland

mmh@ru. is

Thomas Dueholm Hansen

Department of Computer Science, University

of Copenhagen, Denmark

dueholm@di.ku.dk

Sariel Har-Peled

Department of Computer Science, University of Illinois, Urbana, IL, USA

sariel@illinois.edu

Tobias Harks

Universität Augsburg, Institut für

Mathematik, Augsburg, Germany

tobias.harks@math . uni-augsburg.de

Koyo Hayashi

Department of Mathematical Informatics, University of Tokyo, Tokyo 113-8656, Japan koyo_hayashi@mist.i.u-tokyo.ac.jp

Benjamin Hellouin de Menibus

Laboratoire de Recherche en Informatique,

Université Paris-Sud, CNRS,

CentraleSupélec, Université Paris-Saclay,

France

hellouin@lri.fr

https://orcid.org/0000-0001-5194-929X

Danny Hermelin

Ben-Gurion University of the Negev, Beer

Sheva, Israel

hermelin@bgu.ac.il
Niklas Hjuler

University of Copenhagen, Copenhagen,

Denmark

niklashjuler@gmail.com

https://orcid.org/0000-0002-0815-670X

Rani Hod

Department of Mathematics, Bar-Ilan

University, Ramat Gan, Israel

rani.hod@math.biu.ac.il

Martin Hoefer

Goethe-Universität Frankfurt am Main,

Institut für Informatik, Frankfurt am Main,

Germany

mhoefer@cs.uni-frankfurt.de

Piotr Hofman

University of Warsaw, Poland

https ://orcid.org/0000-0001-9866-3723

Jacob Holm

University of Copenhagen, Copenhagen,

Denmark

jaho@di.ku.dk

https://orcid.org/0000-0001-6997-9251

Yinon Horesh

Technion - Israel Institute of Technology,

Haifa, Israel

ynon980@gmail.com

Mathieu Hoyrup

Université de Lorraine, CNRS, Inria, LORIA,

F-54000 Nancy, France

mathieu.hoyrup@inria.fr

Justin Hsu

University College London, London, UK

Sangxia Huang

Sony Mobile Communications, Lund, Sweden

huang. sangxia@gmail.com

Zhiyi Huang

Department of Computer Sicence, The

University of Hong Kong, Hong Kong

zhiyi@cs.hku.hk

Anja Huber

Universität Augsburg, Institut für

Mathematik, Augsburg, Germany

anja.huber@math.uni-augsburg.de 
Pavel Hubáček

Computer Science Institute, Charles

University, Prague, Czech Republic

hubacek@iuuk.mff.cuni.cz

Danny Hucke

Universität Siegen, Germany

hucke@eti.uni-siegen.de

Christoph Hunkenschröder

EPFL, 1015 Lausanne, Switzerland christoph.hunkenschroder@epfl.ch

Russell Impagliazzo

Department of Computer Science, University of California San Diego, La Jolla, CA, USA

russell@cs.ucsd.edu

Piotr Indyk

Department of Computer Science, MIT, Cambridge, MA, USA

indyk@mit.edu

Davis Issac

Max Planck Institute for Informatics, Saarland Informatics Campus, Germany dissac@mpi-inf.mpg.de https://orcid.org/0000-0001-5559-7471

Abhishek Jain

Johns Hopkins University

abhishek@cs.jhu.edu

Jisu Jeong

Department of Mathematical Sciences,

KAIST, Daejeon, Korea

jisujeong89@gmail.com

Mark Jerrum

School of Mathematical Sciences, Queen

Mary, University of London, Mile End Road, London, E1 4NS, United Kingdom

m. jerrum@qmul.ac.uk

https://orcid.org/0000-0003-0863-7279

Timothy Johnson

Dept. of Computer Science, Univ. of

California, Irvine, Irvine, CA 92697 USA

tujohnso@uci.edu

Marcin Jurdziński

University of Warwick, Coventry, UK

Marcin. Jurdzinski@warwick.ac.uk
Sven Jäger

Institut für Mathematik, Technische

Universität Berlin, Germany

jaeger@math.tu-berlin.de

Daniel M. Kane

Department of Computer Science and

Engineering/Department of Mathematics, University of California, San Diego

dakane@ucsd.edu

https://orcid.org/0000-0002-5884-3487

Iyad Kanj

School of Computing, DePaul University,

Chicago, USA

ikanj@cs.depaul.edu

Haim Kaplan

School of Computer Science, Tel Aviv

University, Tel Aviv 69978, Israel

haimk@tau.ac.il

Adam Karczmarz

University of Warsaw, Poland

a. karczmarz@mimuw.edu.pl

Matthew J. Katz

Department of Computer Science,

Ben-Gurion University of the Negev,

Beer-Sheva 84105, Israel

matya@cs.bgu.ac.il

Maximilian Katzmann

Hasso Plattner Institute, University of

Potsdam, Potsdam, Germany

maximilian.katzmann@hpi.de

Ken-ichi Kawarabayashi

National Institute of Informatics, 2-1-2

Hitotsubashi, Chiyoda-ku, Tokyo 101-8430,

Japan

k_keniti@nii.ac.jp

https://orcid.org/0000-0001-6056-4287

Nathan Keller

Department of Mathematics, Bar-Ilan

University, Ramat Gan, Israel

nkeller@math.biu.ac.il

Thomas Kesselheim

University of Bonn, Institute of Computer

Science, Bonn, Germany

thomas.kesselheim@uni-bonn.de 
Stefan Kiefer

University of Oxford, United Kingdom

David H. K. Kim

Computer Science Department, University of Chicago, 1100 East 58th Street, Chicago, Illinois 60637, USA

hongk@cs.uchicago.edu

Eun Jung Kim

Université Paris-Dauphine, PSL Research

University, CNRS, Paris, France

eun-jung.kim@dauphine.fr

Michael P. Kim

Department of Computer Science, Stanford University, Stanford, CA, USA

mpk@cs.stanford.edu

Kim-Manuel Klein

EPFL, 1015 Lausanne, Switzerland

kim-manuel.klein@epfl.ch

Ines Klimann

Univ Paris Diderot, Sorbonne Paris Cité, IRIF, UMR 8243 CNRS, F-75013 Paris,

France

klimann@irif.fr

Max Klimm

School of Business and Economics, HU

Berlin, Spandauer Str. 1, 10099 Berlin,

Germany

max.klimm@hu-berlin.de

https://orcid.org/0000-0002-9061-2267

Sang-Ki Ko

Korea Electronics Technology Institute,

South Korea

sangkiko@keti.re.kr

Bojana Kodric

MPI for Informatics and Saarland University,

Saarbrücken, Germany

bojana@mpi-inf .mpg.de

Zhuan Khye Koh

Department of Combinatorics and

Optimization, University of Waterloo,

Waterloo, Canada

zkkoh@uwaterloo.ca
Alexandra Kolla

Department of Computer Science, University of Colorado at Boulder

alexandra.kolla@colorado.edu

Tsvi Kopelowitz

Bar Ilan University, Ramat Gan, Israel

kopelot@gmail.com

Guy Kortsarz

Rutgers University, Camden, NJ, USA

guyk@camden.rutgers . edu

Martin Koutecký

Technion - Israel Institute of Technology,

Haifa, Israel, and, Charles University,

Prague, Czech Republic

koutecky@technion.ac.il

https://orcid.org/0000-0002-7846-0053

Ioannis Koutis

Department of Computer Science, New

Jersey Institute of Technology

ioannis.koutis@njit.edu

Dariusz R. Kowalski

Computer Science Department, University of

Liverpool, Liverpool, UK

D.Kowalski@liverpool.ac.uk

Jakub Kozik

Theoretical Computer Science Department, Faculty of Mathematics and Computer Science, Jagiellonian University, Kraków, Poland

jakub.kozik@uj.edu.pl

Stephan Kreutzer

Technical University Berlin

stephan.kreutzer@tu-berlin.de

Karel Král

Computer Science Institute, Charles

University, Prague, Czech Republic

kralka@iuuk.mff.cuni.cz

Amit Kumar

IIT Delhi, New Delhi, India

Ananya Kumar

Carnegie Mellon University, Pittsburgh,

United States

skywalker94@gmail.com 
Orna Kupferman

School of Computer Science and Engineering, The Hebrew University, Israel

Dietrich Kuske

Technische Universität Ilmenau, Germany

dietrich.kuske@tu-ilmenau.de

William Kuszmaul

Department of Computer Science, Stanford University, Stanford, CA, USA

kuszmaul@cs.stanford.edu

Karim Labib

Department of Computer Science, ETH

Zürich, Switzerland

labibk@student.ethz.ch

Anissa Lamani

Laboratoire MIS \& Université de Picardie

Jules Verne, Amiens, France

anissa.lamani@u-picardie.fr

Michael Lampis

Université Paris-Dauphine, PSL Research University, CNRS, UMR 7243 , LAMSADE, 75016, Paris, France

michail.lampis@dauphine.fr

Gad M. Landau

University of Haifa, Israel

landau@cs.haifa.ac.il

Harry Lang

Johns Hopkins University, Baltimore, United

States

hlang8@math.jhu.edu

Sławomir Lasota

University of Warsaw

sl@mimuw.edu.pl

https://orcid.org/0000-0001-8674-4470

Ranko Lazić

University of Warwick, Coventry, UK

R.S.Lazic@warwick.ac.uk

Christoph Lenzen

Max Planck Institute for Informatics, Saarbrücken, Germany

clenzen@mpi-inf .mpg.de
Jérôme Leroux

Univ.Bordeaux, CNRS, Bordeaux-INP,

Talence, France

jerome.leroux@labri.fr

Reut Levi

Weizmann Institute of Science, Rehovot, Israel

reut.levi@weizmann.ac.il

https://orcid.org/0000-0003-3167-1766

Asaf Levin

Technion - Israel Institute of Technology, Haifa, Israel

levinas@ie.technion.ac.il

Bo Li

Department of Computer Science, Stony Brook University, Stony Brook, NY 11794, USA

boli2@cs.stonybrook.edu

Jason Li

Carnegie Mellon University

\section{Jian Li}

Institute for Interdisciplinary Information Sciences, Tsinghua University, Beijng, China Correspondingauthorlijian83@mail. tsinghua.edu.cn

Yingkai Li

Department of Computer Science, Stony Brook University, Stony Brook, NY 11794, USA

yingkli@cs.stonybrook.edu

Vahid Liaghat

Facebook, Building 25, 190 Jefferson Dr, Menlo Park, CA 94025, USA

Nutan Limaye

Department of CSE, IIT Bombay, Mumbai, India

nutan@cse.iitb.ac.in

Sixue Liu

Department of Computer Science, Princeton University , 35 Olden Street, Princeton, NJ 08540, USA

sixuel@cs.princeton.edu 
Yang P. Liu

MIT, Cambridge, MA

yangpatil@gmail.com

Markus Lohrey

Universität Siegen, Germany

lohrey@eti.uni-siegen.de

Daniel Lokshtanov

Department of Informatics, University of

Bergen, Norway

daniello@uib.no

Anand Louis

Indian Institute of Science, Bangalore, India

anandl@iisc.ac.in

Shachar Lovett

Department of Computer Science and

Engineering, University of California, San

Diego

slovett@cs.ucsd.edu

https://orcid.org/0000-0003-4552-1443

Pinyan $\mathrm{Lu}$

Institute for Theoretical Computer Science,

Shanghai University of Finance and

Economics, Shanghai 200433, China

lu.pinyan@mail.shufe.edu.cn

Anna Lubiw

School of Computer Science, University of

Waterloo, Canada

alubiw@uwaterloo.ca

Anna Lysyanskaya

Computer Science Department, Brown

University, Providence, RI 02912 USA

anna@cs. brown.edu

Kaifeng Lyu

Institute for Interdisciplinary Information

Sciences, Tsinghua University, Beijing, China

lkf15@mails.tsinghua.edu.cn

Vivek Madan

Department of Computer Science, University

of Illinois, Urbana-Champaign

vmadan2@illinois.edu

Sepideh Mahabadi

Data Science Institute, Columbia University,

New York, NY, USA

mahabadi@mit.edu
Frederik Mallmann-Trenn

CSAIL, MIT, US

mallmann@mit.edu

Nil Mamano

University of California, Irvine, U.S

nmamano@uci.edu

Florin Manea

Department of Computer Science, Kiel

University, D-24098 Kiel, Germany

flm@zs.uni-kiel.de

Pasin Manurangsi

University of California, Berkeley, USA

pasin@berkeley.edu

Yuchen Mao

Department of Computer Science and

Engineering, HKUST, Hong Kong

ymaoad@cse.ust.hk

https://orcid.org/0000-0002-1075-344X

Liran Markin

University of Haifa, Israel

liran.markin@gmail.com

Filip Mazowiecki

Université de Bordeaux, Bordeaux, France filip.mazowiecki@u-bordeaux.fr

Saeed Mehrabi

School of Computer Science, Carleton

University, Ottawa, Canada

saeed.mehrabi@carleton.ca

Ruta Mehta

University of Illinois Urbana-Champaign,

Champaign, USA

rutameht@illinois.edu

George B. Mertzios

Department of Computer Science, Durham

University, UK

George.Mertzios@durham.ac.uk

https://orcid.org/0000-0001-7182-585X

Daniele Miccianco

Department of Computer Science and

Engineering, University of California - San

Diego, CA, USA

daniele@cs.ucsd.edu 
Vahab Mirrokni

Google Research, New York

Pradipta Mitra

Google Research, New York, USA

ppmitra@gmail.com

Matthias Mnich

Universität Bonn, Germany , and Maastricht University, The Netherlands

mmnich@uni-bonn. de

https://orcid.org/0000-0002-4721-5354

Sidhanth Mohanty

Computer Science Department, Carnegie

Mellon University, Pittsburgh, PA, USA

sidhanth@cmu.edu

Marco Molinaro

PUC-Rio, Rio de Janeiro, Brazil

molinaro@inf.puc-rio.br

Fabrizio Montecchiani

Department of Engineering, University of Perugia, Perugia, Italy

fabrizio.montecchiani@unipg.it

Felix Montenegro-Retana

Hasso Plattner Institute, University of

Potsdam, Potsdam, Germany

felix.montenegro-retana@student.hpi .

de

Shay Moran

Institute for Advanced Study, Princeton

shaymoran@ias.edu

https://orcid.org/0000-0002-8662-2737

Pat Morin

School of Computer Science, Carleton

University, Ottawa, Canada

morin@scs.carleton.ca

Hagar Mosaad

Department of Computer Science and

Engineering, German University in Cairo,

Egypt

hagar.omar@student.guc.edu.eg

Luca Moscardelli

Department of Economic Studies, University of Chieti-Pescara, Pescara, Italy

luca.moscardelli@unich.it
Miguel A. Mosteiro

Computer Science Department, Pace

University, New York, NY, USA

mmosteiro@pace.edu

Anish Mukherjee

Chennai Mathematical Institute, Chennai, India

anish@cmi.ac.in

Cameron Musco

CSAIL, MIT, US

cnmusco@mit.edu

Christopher Musco

CSAIL, MIT, US

cpmusco@mit.edu

Torsten Mütze

Institut für Mathematik, Technische

Universität Berlin, Germany

muetze@math.tu-berlin.de

Hiroshi Nagamochi

Department of Applied Mathematics and Physics, Graduate School of Informatics, Kyoto University, Japan

nag@amp.i.kyoto-u.ac.jp

Vasileios Nakos

Harvard University, Cambridge, USA

vasileiosnakos@g.harvard.edu

Maryam Negahbani

Department of Computer Science,

Dartmouth College, 9 Maynard St, Hanover, NH, USA

maryam@cs.dartmouth.edu

Yakov Nekrich

Cheriton School of Computer Science,

University of Waterloo, Canada

yakov.nekrich@googlemail.com

Daniel Neuen

RWTH Aachen University, Aachen, Germany

neuen@informatik.rwth-aachen.de

Jaroslav Nešetřil

Computer Science Institute, Charles

University, Prague, Czech Republic

nesetril@iuuk.mff.cuni.cz 
Rachit Nimavat

Toyota Technological Institute at Chicago,

6045 S. Kenwood Ave., Chicago, Illinois

60637, USA

nimavat@ttic.edu

Chinmay Nirkhe

Electrical Engineering and Computer

Sciences, University of California, Berkeley ,

387 Soda Hall Berkeley, CA 94720, U.S.A

nirkhe@cs.berkeley.edu

Reino Niskanen

Department of Computer Science, University of Liverpool, UK

r.niskanen@liverpool.ac.uk

Dirk Nowotka

Department of Computer Science, Kiel University, D-24098 Kiel, Germany

dn@zs.uni-kiel.de

Marc Noy

Universitat Politècnica de Catalunya,

Barcelona,marc.noy@upc.edu

Krzysztof Onak

IBM Research, TJ Watson Research Center, Yorktown Heights, New York, USA

Shmuel Onn

Technion - Israel Institute of Technology,

Haifa, Israel

onn@ie.technion.ac.il

Patrice Ossona de Mendez

CAMS (CNRS, UMR 8557), Paris, France

Rafail Ostrovsky

Department of Computer Science, University of California Los Angeles, USA

rafail@cs.ucla.edu

Joël Ouaknine

Max Planck Institute for Software Systems, Germany \& , Department of Computer Science, Oxford University, UK

joel@mpi-sws.org

Sang-il Oum

Department of Mathematical Sciences,

KAIST, Daejeon, Korea

sangil@kaist.edu
Katarzyna Paluch

Wroclaw University, Poland

abraka@cs.uni.wroc.pl

https://orcid.org/0000-0002-7504-6340

Fahad Panolan

Department of Informatics, University of

Bergen, Norway

Fahad.Panolan@uib.no

https ://orcid.org/0000-0001-6213-8687

Charles Paperman

Université de Lille

Merav Parter

Weizmann IS, Rehovot, Israel

merav.parter@weizmann.ac.il

Sarvar Patel

Google LLC, Mountain View, USA

sarvar@google.com

Boaz Patt-Shamir

Tel Aviv University, Tel Aviv, Israel

boaz@tau.ac.il

John Peebles

CSAIL,MIT, Cambridge, MA, USA

jpeebles@mit.edu

Giuseppe Persiano

Google LLC, Mountain View, USA and

Università di Salerno, Salerno, Italy

giuper@gmail.com

Michał Pilipczuk

University of Warsaw, Warsaw, Poland

Thomas Place

LaBRI, University of Bordeaux and IUF,

France

Ely Porat

Bar Ilan University, Ramat Gan, Israel

porately@cs.biu.ac.il

Igor Potapov

Department of Computer Science, University of Liverpool, UK

potapov@liverpool.ac.uk

Manoj Prabhakaran

Indian Institute of Technology Bombay

mp@cse.iitb.ac.in 
Eric Price

UT Austin, Austin, TX, USA

ecprice@cs.utexas.edu

Gabriele Puppis

CNRS, LaBRI, Université de Bordeaux

Guillermo A. Pérez

Université libre de Bruxelles, Brussels,

Belgium

gperezme@ulb.ac.be

https://orcid.org/0000-0002-1200-4952

Yuval Rabani

The Rachel and Selim Benin School of Computer Science and Engineering, The Hebrew University of Jerusalem, Israel yrabani@cs.huji.ac.il

Saladi Rahul

Dept. of Computer Science, University of Illinois at Urbana-Champaign, USA

saladi@uiuc.edu

M. S. Ramanujan

Algorithms and Complexity Group, TU

Wien, Austria

ramanujan@ac.tuwien.ac.at

M. S. Ramanujan

University of Warwick, United Kingdom

R.Maadapuzhi-Sridharan@warwick.ac.uk

Sofya Raskhodnikova

Boston University, Boston, USA

sofya@bu.edu

Mikhail Raskin

LaBRI, University of Bordeaux, 351, cours de la Libération F-33405 Talence cedex, France

raskin@mccme.ru

https: //orcid.org/0000-0002-6660-5673

Gaurav Rattan

RWTH Aachen University, Aachen, Germany grohe@informatik.rwth-aachen. de https://orcid.org/0000-0002-5095-860X
Alexander Ravsky

Pidstryhach Institute for Applied Problems of Mechanics and Mathematics, National Academy of Science of Ukraine, Lviv, Ukraine

oravsky@mail.ru

Felix Reidl

Royal Holloway, University of London,

TW20 0EX, UK

Felix.Reidl@rhul.ac.uk

Hanlin Ren

Institute for Interdisciplinary Information Sciences, Tsinghua University, China

rhl16@mails.tsinghua.edu.cn

Michael Riabzev

Technion - Israel Institute of Technology, Haifa, Israel

riabzevmichael@gmail.com

Sören Riechers

Paderborn University, Paderborn, Germany

Andrei Romashchenko

LIRMM, Univ Montpellier, CNRS,

Montpellier, France; on leave from IITP RAS

andrei.romashchenko@lirmm.fr

\section{Eyal Ronen}

Computer Science Department, The

Weizmann Institute, Rehovot, Israel

eyal.ronen@weizmann.ac.il

Eva Rotenberg

Technical University of Denmark, Lyngby,

Denmark

erot@dtu.dk

https://orcid.org/0000-0001-5853-7909

Ron D. Rothblum

MIT and Northeastern University,

Cambridge, MA

ronr@mit.edu

Tim Roughgarden

Department of Computer Science, Stanford

University, Stanford, CA 94305, USA

Daniel M. Roy

Univ. Toronto 
Clemens Rösner

Department of Theoretical Computer

Science, University of Bonn, Germany

roesner@cs.uni-bonn.de

Aleksi Saarela

Department of Mathematics and Statistics,

University of Turku, 20014 Turku, Finland

amsaar@utu.fi

https://orcid.org/0000-0002-6636-2317

\author{
Manuel Sabin \\ Computer Science Division, University of \\ California Berkeley, Berkeley, CA, USA \\ msabin@berkeley . edu
}

Eden Saig

Department of Computer Science, Technion,

Haifa, Israel

edens@cs.technion.ac.il

https://orcid.org/0000-0002-0810-2218

Laura Sanità

Department of Combinatorics and

Optimization, University of Waterloo,

Waterloo, Canada

Isanita@uwaterloo.ca

Piotr Sankowski

Institute of Informatics, University of

Warsaw

sank@mimuw.edu.pl

Diego Nava Saucedo

Université de Lorraine, CNRS, Inria, LORIA,

F-54000 Nancy, France

diego.nava-saucedo@wanadoo.fr

Saket Saurabh

Institute of Mathematical Sciences, HBNI,

India and UMI ReLax

saket@imsc.res.in

Rahul Savani

University of Liverpool, UK

rahul.savani@liverpool.ac.uk

Joe Sawada

School of Computer Science, University of

Guelph, Canada

jsawada@uoguelph.ca
Nitin Saxena

Department of Computer Science, IIT

Kanpur, India

nitin@cse.iitk.ac.in

Marco Scarsini

Dipartimento di Economia e Finanza, LUISS,

Viale Romania 32, 00197 Roma, Italy

marco.scarsini@luiss.it

https://orcid.org/0000-0001-6473-794X

Baruch Schieber

IBM Research, TJ Watson Research Center, Yorktown Heights, New York, USA

Andreas Schmid

Max Planck Institute for Informatics,

Saarbrücken, Germany

aschmid@mpi-inf .mpg.de

Stefan Schmid

University of Vienna, Austria

stefan_schmid@univie.ac.at

Jens M. Schmidt

Technische Universität Ilmenau, Ilmenau,

Germany

jens.schmidt@tu-ilmenau.de

Melanie Schmidt

Department of Theoretical Computer

Science, University of Bonn, Germany

melanieschmidt@uni-bonn.de

Alexander A. Schwarzmann

University of Connecticut, Storrs CT, USA

ass@uconn.edu

Nicole Schweikardt

Humboldt-Universität zu Berlin, Germany

schweikn@informatik.hu-berlin.de

Pascal Schweitzer

Technische Universität Kaiserslautern,

Kaiserslautern, Germany

schweitzer@cs.uni-kl.de

Saeed Seddighin

University of Maryland, College Park, MD

20742, USA

C. Seshadhri

Department of Computer Science, University of California, Santa Cruz, CA 95064, USA 
Amirbehshad Shahrasbi

Carnegie Mellon University, Pittsburgh, PA, USA

shahrasbi@cs.cmu.edu

Jeffrey Shallit

School of Computer Science, University of Waterloo, Canada

shallit@cs.uwaterloo.ca

Adi Shamir

Computer Science Department, The

Weizmann Institute, Rehovot, Israel

adi.shamir@weizmann.ac.il

Micha Sharir

School of Computer Science, Tel Aviv

University, Tel Aviv 69978, Israel

michas@tau.ac.il

Roohani Sharma

Institute of Mathematical Sciences, HBNI, India and UMI ReLax

roohani@imsc.res.in

Xiaofei Shi

Carnegie Mellon University, Pittsburgh, USA

xiaofeis@andrew.cmu.edu

Philip Shilane

Dell EMC, Newtown, PA, USA

shilane@dell.com

Mahsa Shirmohammadi

CNRS \& LIS, France

mahsa.shirmohammadi@lis-lab.fr

Sebastian Siebertz

University of Warsaw, Warsaw, Poland

Alexandra Silva

University College London, London, UK

Luis Fernando Schultz Xavier da Silveira School of Computer Science and Electrical Engineering, University of Ottawa, Ottawa, Canada

schultz@ime.usp.br

Aditi Singh

IIT Gandhinagar, Gandhinagar, India aditi.singh@iitgn.ac.in
Ali Kemal Sinop

TOBB University of Economics and

Technology, Ankara, Turkey

asinop@gmail.com

Piotr Skowron

University of Warsaw, Warsaw, Poland

p.skowron@mimuw.edu.pl

Michał Skrzypczak

University of Warsaw, Banacha 2, 02-097

Warsaw, Poland

mskrzypczak@mimuw . edu .pl

https://orcid.org/0000-0002-9647-4993

Veronika Slívová

Computer Science Institute, Charles

University, Prague, Czech Republic

slivova@iuuk.mff.cuni.cz

Shay Solomon

IBM Research, T. J. Watson Research

Center, Yorktown Heights, New York, USA

Tasuku Soma

The University of Tokyo, Tokyo, Japan

tasuku_soma@mist.i.u-tokyo.ac.jp

https://orcid.org/0000-0001-9519-2487

Manuel Sorge

Ben-Gurion University of the Negev, Beer

Sheva, Israel

sorge@post.bgu.ac.il

Krzysztof Sornat

University of Wrocław, Wrocław, Poland

krzysztof .sornat@cs.uni.wroc.pl

Jessica Sorrell

Department of Computer Science and

Engineering, University of California, San

Diego, CA, USA

jlsorrel@cs.ucsd.edu

Paul G. Spirakis

Department of Computer Science, University

of Liverpool, UK, Department of Computer

Engineering \& Informatics, University of

Patras, Greece

P.Spirakis@liverpool.ac.uk

https://orcid.org/0000-0001-5396-3749 
Joachim Spoerhase

Lehrstuhl für Informatik I, Universität

Würzburg, Germany and Institute of

Computer Science, University of Wrocław,

Poland

joachim.spoerhase@uni-wuerzburg

https://orcid.org/0000-0002-2601-6452

Srikanth Srinivasan

Department of Mathematics, IIT Bombay,

Mumbai, India

srikanth@math.iitb.ac.in

Sam Staton

Department of Computer Science, University of Oxford, Oxford OX1 3QD UK

sam.staton@cs.ox.ac.uk

Cliff Stein

Columbia University, New York, USA

Dario Stein

Univ. Oxford

Damian Straszak

École Polytechnique Fédérale de Lausanne

(EPFL), Switzerland

Don M. Stull

Université de Lorraine, CNRS, Inria, LORIA, F-54000 Nancy, France

donald.stull@inria.fr

Madhu Sudan

Harvard University, Cambridge, MA, USA

madhu@cs.harvard.edu

Wing-Kin Sung

National University of Singapore, Singapore

ksung@comp.nus.edu.sg

Manuel Surek

Universität Augsburg, Institut für

Mathematik, Augsburg, Germany

manuel.surek@math.uni-augsburg. de

Ola Svensson

École Polytechnique Fédérale de Lausanne,

Lausanne, Switzerland

ola.svensson@epfl.ch
Mani Swaminathan

Department of Computing Science,

University of Oldenburg, Germany

mani.swaminathan@informatik.

uni-oldenburg.de

Chaitanya Swamy

Dept. of Combinatorics and Optimization,

Univ. Waterloo, Waterloo, ON N2L 3G1,

Canada

cswamy@uwaterloo.ca

Géraud Sénizergues

LABRI, Bordeaux, France

geraud.senizergues@u-bordeaux.fr

Zhihao Gavin Tang

Department of Computer Sicence, The

University of Hong Kong, Hong Kong

zhtang@cs.hku.hk

Marianne Thieffry

Hasso Plattner Institute, University of

Potsdam, Potsdam, Germany

marianne.thieffry@student.hpi.de

Thomas Thierauf

Aalen University, Germany

Mikkel Thorup

BARC, University of Copenhagen,

Universitetsparken 1, Copenhagen, Denmark

mikkel2thorup@gmail.com

https://orcid.org/0000-0001-5237-1709

Tigran Tonoyan

School of Computer Science, Reykjavik

University, Iceland

ttonoyan@gmail.com

Szymon Toruńczyk

University of Warsaw, Warsaw, Poland

Konstantinos Tsakalidis

Dept. of Computer and Information Science,

Tandon School of Engineering, New York

University, USA

kt79@nyu.edu

Eli Upfal

Computer Science Department, Brown

University, Providence, RI 02912 USA

eli@cs.brown.edu 
Przemysław Uznański

Department of Computer Science, ETH

Zürich, Switzerland

przemyslaw.uznanski@inf .ethz.ch

Pascal Vanier

Laboratoire d'Algorithmique, Complexité et

Logique, Université Paris-Est Créteil, France

pascal. vanier@lacl.fr

Gal Vardi

School of Computer Science and Engineering,

The Hebrew University, Israel

Shai Vardi

California Institute of Technology, Pasadena

CA, USA

svardi@caltech.edu

Nithin Varma

Boston University, Boston, USA

nvarma@bu.edu

Yadu Vasudev

Indian Institute of Technology Madras,

Chennai, India

yadu@cse.iitm.ac.in

https://orcid.org/0000-0001-7918-7194

Umesh Vazirani

Electrical Engineering and Computer

Sciences, University of California, Berkeley , 387 Soda Hall Berkeley, CA 94720, U.S.A

vazirani@cs.berkeley.edu

Rakesh Venkat

Hebrew University of Jerusalem, Israel

rakesh@cs.huji.ac.il

Suresh Venkatasubramanian

School of Computing, University of Utah, USA

suresh@cs.utah.edu

Cosimo Vinci

Department of Information Engineering

Computer Science and Mathematics,

University of L'Aquila, L'Aquila, Italy -

Gran Sasso Science Institute, L'Aquila, Italy

cosimo.vinci@univaq.it

Emanuele Viola

Northeastern University

viola@ccs.neu.edu
Nisheeth K. Vishnoi

École Polytechnique Fédérale de Lausanne

(EPFL), Switzerland

Ellen Vitercik

Carnegie Mellon University, Pittsburgh, PA, USA

vitercik@cs.cmu.edu

Nils Vortmeier

TU Dortmund University, Dortmund,

Germany

nils.vortmeier@tu-dortmund.de

Magnus Wahlström

Royal Holloway, University of London,

TW20 0EX, UK

Magnus.Wahlstrom@rhul.ac.uk

David Wajc

Carnegie Mellon University, Pittsburgh, USA

Stefan Walzer

Technische Universität Ilmenau, Germany

stefan.walzer@tu-ilmenau.de

https://orcid.org/0000-0002-6477-0106

Fan Wei

Department of Mathematics, Stanford

University, Stanford, CA 94305, USA

Oren Weimann

University of Haifa, Israel

oren@cs.haifa.ac.il

Nicole Wein

EECS, Massachusetts Institute of

Technology, Cambridge, MA 02139, USA

Armin Weiß

Universität Stuttgart, FMI, Germany

armin.weiss@fmi.uni-stuttgart.de

Daniel Wiebking

RWTH Aachen University, Aachen, Germany

wiebking@informatik.rwth-aachen.de

Sebastian Wiederrecht

TU Berlin, Germany

sebastian.wiederrecht@tu-berlin.de 
Kaja Wille

Institut für Mathematik, Technische

Universität Berlin, Germany

wille@math.tu-berlin.de

Richard Ryan Williams

MIT EECS and CSAIL, 32 Vassar St,

Cambridge, MA 02139 USA

rrw@mit.edu

https://orcid.org/0000-0003-2326-2233

Sarah Winter

RWTH Aachen University, Germany

winter@cs.rwth-aachen.de

Felix Wolf

Technische Universität Darmstadt, Institute

TEMF, Graduate School of Excellence

Computational Engineering

wolf@gsc.tu-darmstadt.de

David P. Woodruff

Carnegie Mellon University, School of

Computing, Pittsburg, USA

dwoodruf@cs . cmu . edu

James Worrell

University of Oxford, Oxford, UK

James. Worrell@cs . ox . ac . uk

https://orcid.org/0000-0001-8151-2443

Xiaowei $\mathrm{Wu}$

Department of Computing, The Hong Kong

Polytechnic University, Hong Kong

wxw0711@gmail.com

Maximilian Wötzel

BGSMath and UPC Barcelona, Barcelona, Spain

maximilian. wotzel@upc.edu

https://orcid.org/0000-0001-7591-0998

Mingyu Xiao

School of Computer Science and Engineering,

University of Electronic Science and

Technology of China, Chengdu, China

myxiao@gmail.com

https://orcid.org/0000-0002-1012-2373

Yuanhang Xie

Institute for Interdisciplinary Information

Sciences, Tsinghua University, Beijing, China

xieyh15@mails.tsinghua.edu.cn
Pan Xu

Department of Computer Science, University of Maryland, College Park, USA

panxu@cs.umd.edu

Hongseok Yang

KAIST

Lin F. Yang

Princeton University, Princeton, United

States

lin.yang@princeton.edu

Harel Yedidsion

Ben-Gurion University of the Negev, Beer

Sheva, Israel

yedidsio@post.bgu.ac.il

Kevin Yeo

Google LLC, Mountain View, USA

kwlyeo@google.com

Yuichi Yoshida

National Institute of Informatics, Preferred Infrastructure, Tokyo, Japan

yyoshida@nii.ac.jp

https://orcid.org/0000-0001-8919-8479

Arman Yousefi

Department of Computer Science, University of California Los Angeles, USA

armany@cs.ucla.edu

Ching-Hua $\mathrm{Yu}$

University of Illinois at Urbana-Champaign

cyu17@illinois.edu

Henry Yuen

Electrical Engineering and Computer

Sciences, University of California, Berkeley , 387 Soda Hall Berkeley, CA 94720, U.S.A

hyuen@cs.berkeley.edu

Viktor Zamaraev

Department of Computer Science, Durham

University, UK

Viktor.Zamaraev@durham.ac.uk

https://orcid.org/0000-0001-5755-4141

Meirav Zehavi

Department of Computer Science,

Ben-Gurion University, Israel

meiravze@bgu.ac.il 
Marc Zeitoun

LaBRI, University of Bordeaux, France

Georg Zetzsche

IRIF (Université Paris-Diderot \& CNRS),

France

https://orcid.org/0000-0002-6421-4388

Thomas Zeume

TU Dortmund University, Dortmund,

Germany

thomas.zeume@tu-dortmund.de

Hongyang Zhang

Carnegie Mellon University, Pittsburgh, USA

hongyanz@cs.cmu .edu

Le Zhang

Institute for Interdisciplinary Information Sciences, Tsinghua University, Beijing, China le-zhang12@mails.tsinghua.edu.cn

Yuhao Zhang

Department of Computer Sicence, The University of Hong Kong, Hong Kong

yhzhang2@cs.hku.hk

Zeyu Zhang

Johns Hopkins University, Baltimore, MD, USA

zyzhang92@gmail.com

Samson Zhou

Department of Computer Science, Purdue University, West Lafayette, Indiana, USA

samsonzhou@gmail.com

Marius Zimand

Department of Computer and Information

Sciences, Towson University, Baltimore, MD 
